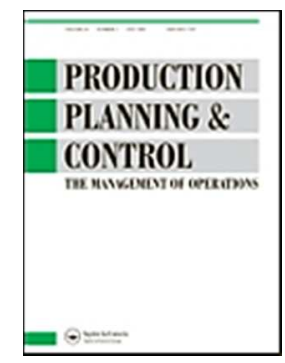

\title{
Modeling activity times by hybrid synthetic method
}

\begin{tabular}{|r|l|}
\hline Journal: & Production Planning \& Control \\
\hline Manuscript ID & TPPC-2015-0050.R3 \\
\hline Manuscript Type: & Research \\
\hline Complete List of Authors: & $\begin{array}{l}\text { Lanzetta, Michele; University of Pisa, Department of Civil and Industrial } \\
\text { Engineering } \\
\text { Rossi, Andrea; University of Pisa, Department of Civil and Industrial } \\
\text { Engineering } \\
\text { Puppato, Alessio; Azienda Ospedaliero-Universitaria Pisana } \\
\text { Pagliantini, Silvia; Azienda Ospedaliero-Universitaria Pisana }\end{array}$ \\
\hline Keywords: & $\begin{array}{l}\text { Uncertain time, Manual production, Process standardization, Operator } \\
\text { efficiency, Healthcare }\end{array}$ \\
\hline
\end{tabular}

\section{SCHOLARONE ${ }^{\text {m }}$}

Manuscripts 


\title{
Modeling activity times by hybrid synthetic method
}

\author{
Michele LANZETTA \\ Department of Civil and Industrial Engineering, University of Pisa \\ lanzetta@unipi.it \\ Andrea ROSSI \\ Department of Civil and Industrial Engineering, University of Pisa \\ andrea.rossi1@unipi.it
}

\author{
Alessio PUPPATO \\ Azienda Ospedaliero-Universitaria Pisana \\ a.puppato@ao-pisa.toscana.it \\ Silvia PAGLIANTINI \\ Azienda Ospedaliero-Universitaria Pisana \\ s.pagliantini@ao-pisa.toscana.it
}




\title{
Modeling activity times by hybrid synthetic method
}

\begin{abstract}
Uncertain (manual) activity times impact a number of manufacturing system modules: plant and layout design, capacity analysis, operator assignment, process planning, scheduling and simulation. Direct observation cannot be used for non-existent production lines. A hybrid direct observation/synthetic method derived from Method Time Measurement MTM available in industry is proposed. To determine accurate activity times required by heuristics and metaheuristics optimization, manufacturing system modules are modeled by MILP and operator efficiency parameters are used for time standardization. Among human factors considered are skill and ergonomics. Application to the sterilization of reusable medical devices is extensively described. Experimental data taken from observation on the field and a worst case date have shown the model direct applicability for professionals also to nonmanufacturing cases.
\end{abstract}

Keywords: Uncertain time, Manual production, Process standardization, Operator efficiency, Healthcare 


\title{
Modeling activity times by hybrid synthetic method
}

\author{
Michele Lanzetta (1anzetta@unipi.it), Andrea Rossi \\ Department of Civil and Industrial Engineering, University of Pisa
}

\author{
Alessio Puppato, Silvia Pagliantini \\ Azienda Ospedaliero-Universitaria Pisana
}

\begin{abstract}
Uncertain (manual) activity times impact a number of manufacturing system modules: plant and layout design, capacity analysis, operator assignment, process planning, scheduling and simulation. Direct observation cannot be used for non-existent production lines. A hybrid direct observation/synthetic method derived from Method Time Measurement MTM available in industry is proposed. To determine accurate activity times required by heuristics and metaheuristics optimization, manufacturing system modules are modeled by MILP and operator efficiency parameters are used for time standardization. Among human factors considered are skill and ergonomics. Application to the sterilization of reusable medical devices is extensively described. Experimental data taken from observation on the field and a worst case date have shown the model direct applicability for professionals also to nonmanufacturing cases.
\end{abstract}

Keywords: Uncertain time, Manual production, Process standardization, Operator efficiency, Healthcare 


\section{Introduction}

When manual operations are concerned, production planning and control requires the definition of activity times, which are affected by the capabilities of individual operators depending on skill, motivation, training and work conditions, like environmental noise, repetitive work, use of force, environmental temperature and light, affecting efficiency.

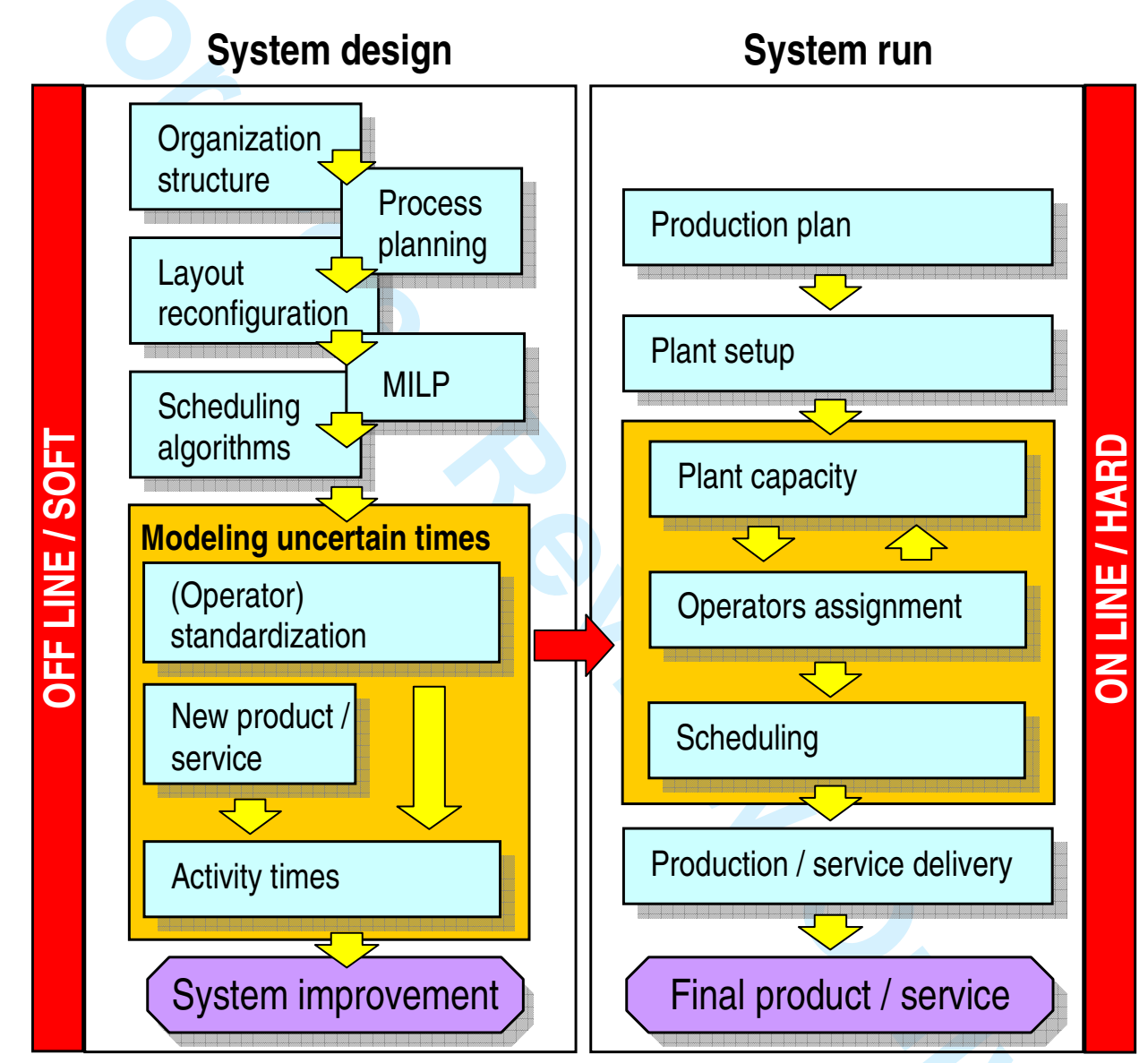

Figure 1 Influence of uncertain activity times in production planning and control.

Activity times affect a large part of the system design and run (Buxey, Slack, and Wild 1973).

Figure 1 shows a typical production planning and control scheme. The shaded areas enhance the connection between the off-line determination of (uncertain) activity times and their use in 
the system run. The system modules represent also non industrial organizations, including immaterial products, like service delivery, where human factors are a dominating aspect.

As for the impact of (uncertain) activity times in offline and online system management, the activity times of manual operations are first defined at the process planning stage. Process planning was defined by the Society of Manufacturing Engineers as the systematic determination of the methods by which a product is to be manufactured economically and competitively. The process plan is a document including an ordered list of (manual and machine) operations (e.g. Table 1), which includes the following information:

- the sequence of stages (routing),

- a list of operations, to be detailed in the operator instruction sheet,

- the activity time (which can be converted to costs where cost drivers are available),

- any additional useful information, like raw materials, tools, machine configuration and drawings.

The process plan requires the definition of activity times for manual operations and can be aggregated as macro operations. The decomposition of operations into sub operations may produce better scheduling, unless the uncertainty or high dispersion of actual times frustrates the intent. The composition/decomposition of operations and the connections between planning and scheduling with flexible (manual) resources were developed in Rossi, Soldani and Lanzetta (2015).

The process plan determines the system layout for a new plant or the new configuration, where necessary, for a new product on an existing plant. The presence of buffers and other constraints are determined at this step and affect the plant model, which can be described as a mixed integer linear programming model (MILP), which allows plant setup, capacity analysis, operator assignment and scheduling. 
Focusing on activity times in scheduling, models in the literature generally assume that all the problem data are known in advance. In reality, though, there can be uncertainty in processing times of manual operations, thus, the solutions generated may not be accurate (Balasubramanian and Grossmann 2002; Aytug et al. 2005). Heuristics to approach the problem are mainly based on stochastic processing times by means of statistical distributions (Ke and Liu 2005, Choi and Wang 2012). Although probability theory has been successfully applied to scheduling problem to tackle the statistical uncertainties, very often the activity time (and cost) cannot be described as a random number with defined probability density function. For example, for new activities, activities without sufficient statistical data and/or those taking into account a number of variables, such as skill, motivation and activity type, the probability distributions for costs and times of activities may be unknown or only partially known. Therefore, use of probabilistic approaches for examining uncertainties in cost and execution time of an activity may not be the appropriate choice. In fact, deficiency (if not lack) of data for an activity in a new environment imposes a sever constraint on the probabilistic approaches (Eshtehardian, Afshar and Abbasnia 2009).

This work is motivated by a concrete problem in healthcare, which is considered in the literature section below: the sterilization of reusable medical devices (RMDs). A delayed delivery may cause the cancellation of surgery!

Current application, extensively discussed in this paper, was introduced by the authors in Rossi, Puppato and Lanzetta (2013). They modeled the examined hospital sterilization plant by analogy to manufacturing as a hybrid (or flexible) flow shop environment, with sequenceindependent prefixed setup times and related parallel batching machines per stage. Manual operations preceding each of the two stages were dealt with as machine setup with fixed times, which were the input of the scheduling problem. In this work we explore the sources of variability of manual setup operation time, and propose a model and MILP of the operator behavior, introducing a set of efficiency parameters $E_{k}$. Efficiency parameters allow taking 
into account the variability in the operator performance. Not only ergonomics are considered, but also skill, stress, motivation and product being handled etc. They are defined in order to standardize setup times, which vary by operator and handled product, and are used in capacity analysis, operator assignment, layout design, scheduling, simulation and performance assessment. These parameters are included in a mixed integer linear programming model to show their relationship with the system design and run. The proposed approach is a hybrid of direct observation and synthetic methods; it is based on Method Time Machine (MTM), recently revised for a number of applications (Bedny, Karwowski and Voskoboynikov 2015, Kuo and Wang 2009). The result of estimation methods is a stochastic distribution; with direct observation, the result is a fixed average value; with the proposed synthetic method, uncertainty is controlled by fixed efficiency parameters. In the proposed MILP these parameters are bound to the different activities for the system optimization.

\section{Literature}

\section{Literature on uncertain times}

This section outlines the main approaches available in the literature to deal with uncertain times. Three main groups of methods are used to determine times for manual activities: estimation, direct observation and synthetic methods. Estimation methods involve different types and uses of statistical distributions. Direct observation methods (the stopwatch) are more expensive and time consuming; besides, direct methods can be applied only for existing production lines. Synthetic methods, descending from the Method Time Measurement, are able to determine appropriate times for manual activities by derivation from preset standards of time for various conditions and performance of operators.

Generally, when estimation methods are concerned, simulations with stochastic times are 
considered. Simulation software is used to evaluate some statistics (minimum, average, maximum, standard deviation) of the selected performance index (Rossi 2014). Gourgand, Grangeon and Norre (2003) proposed a recursive discrete event simulation model based on a Markov chain to evaluate the expected makespan in stochastic permutation flow shop scheduling problem with unlimited buffers. The processing time of each job on each machine was a random variable exponentially distributed with a known rate. Hao, Lin and Gen (2014) proposed an approach to the stochastic resource constrained project scheduling problem with the uncertainty of durations where the aim is to minimize the expected average makespan and expected slack based robustness. They assumed that the probability distribution of the activity duration (uniform distribution) is known in advance. Ahmadizar, Ghazanfari and Fatemi Ghomi (2010) considered the stochastic group shop scheduling problem with the objective to minimize the makespan where both release dates and processing times are random variables, normally, exponentially or uniformly distributed. The problem is formulated in a form of stochastic programming and solved by an optimization approach (ant colony algorithm). Kalhor, Khanzadi, Eshtehardian and Afshar (2011) proposed an ant colony approach to solve the stochastic time-cost tradeoff optimization problem, where uncertainties in time and cost of the project are taken into account. Swaminathan, Pfund, Fowler, Mason and Keha (2007) examined minimization of total weighted tardiness in a dynamic flow shop with uncertain processing times, where new jobs arrive at every shift change. Shift-based scheduling yields competitive results with respect to the approach with dispatching rules, even though dispatching has the advantage of a non-permutation flow shop scheduling (full relaxation of the permutation, as in Rossi and Lanzetta 2014). Arnaout (2014) considered predictive and reactive rescheduling for the unrelated parallel machine problem with sequence dependent setup times and different rates of breakdowns or urgent jobs arrivals. The job processing and setup times are stochastic following uniform distributions. As these values are not known until the job is actually started on the machine, they refine by rescheduling, if the actual setup 
times exceed those considered in the simulation, making their algorithm quite robust. Uniform distributions are considered when high variances of processing times are concerned, to ensure that heuristics are tested under unfavorable conditions. Fuzzy theory was also used to model and examine the consequences of the duration uncertainties (Hapke, Jaszkiewicz and Slowinski 1994). Petrovic and Song (2006) presented an algorithm to minimize the makespan in a two-machine flow shop with triangular fuzzy processing times. Eshtehardian, Afshar and Abbasnia (2009) proposed a multi-objective genetic algorithm in an uncertain environment where fuzzy numbers are used to address the uncertainties and possible variations in the execution time and cost of activities for solving the multiobjective fuzzy time-cost model.

Razmi and Shakhs-Niyaee (2008) presented a type of synthetic methods, the predetermined motion time systems, to determine assembly cycle times in a car industry. This method includes work measurement techniques where times established for basic human motions are used to build up operation times at defined performance levels. Bedny, Karwowski and Voskoboynikov (2015) present standardized language for description of motor components of activity that can be used for creation of models of work activity, which are necessary for ergonomic designs.

It seems that no application of synthetic methods is present in healthcare in order to consider ergonomics for the standardization of operator times.

\section{Literature on hospital sterilization}

The purpose of this section is twofold: show the relevance of the specific healthcare problem and the novelty of the proposed approach, although the proposed operator model is not specific for healthcare, in fact, similar time standardization needs are available in other empirical works on sterilization (Ozturk, Espinouse, Di Mascolo and Gouin 2010), surgery 
(Larsson 2013) and in the car industry (Razmi and Shakhs-Niyaee 2008).

Reymondon, Pellet and Marcon (2008) considered a related problem of tray composition with the objective of minimizing reprocessing and storage costs. They proposed a mathematical programming formulation for the problem of reusable medical devices grouping into packages, which optimizes an objective function composed of storage costs and process costs and discussed computational challenges arising from the problem's size. The main challenge when looking for the best solution of medical device packaging is related to the explosive complexity of the solutions. The problem of packaging medical devices can be classified into the category of partitioning or grouping problems under certain conditions. In the literature, the partitioning problem is considered NP-hard (e.g. Garey and Johnson 2000).

Van de Klundert, Muls and Schadd (2008) considered the cost reduction, when redesigning the sterilization process. Authors were concerned with transportation cost, operating room storage cost, and medical device cost, which, in their opinion, are the three major costs when outsourcing sterilization activities. Using integer linear programming (ILP), they optimized the composition of medical device containers, storage capacity, and delivery time.

Ozturk, Espinouse, Di Mascolo, and Gouin (2012) aimed to minimize the total duration of washing. The problem was studied as a batch scheduling problem where medical devices used for a surgical operation are considered as sets, which may have different sizes and different release dates. They provided and experimented a mixed integer linear programming model (MILP) together with some heuristics based on classical bin packing algorithms.

Di Mascolo and Gouin (2013) presented a discrete event simulation for a generic model of a sterilization service. The model can be used to improve the performance of a specific sterilization service and/or to plan the capacity of its resources. They applied this model to assess and to compare the performance of nine sterilization services and show the advantage 
of such a tool in obtaining performance parameters that are difficult to estimate by the sterilization service decision makers. Furthermore, they quantified the possible improvements obtained by changing some organizational aspects. These changes can be related, for example, to the loading policies for washers or autoclaves, to the staff schedule, or to the opening time of the service. Decision-makers in sterilization services are usually more aware and concerned with quality, safety and traceability aspects than with organizational aspects, which are considered in this work.

Ozturk, Begen and Zaric (2014) considered the parallel batching of jobs on identical machines, considering the makespan as the objective function, in a single step of the flow shop, the washing step, which is a bottleneck for sterilization services, as confirmed in the examined case. The method exploits the structural properties of the problem, propose a search tree to develop an exact method, a branch and bound based algorithm (B\&B). However, they do not consider setup times before bottleneck operations.

Rossi, Puppato and Lanzetta (2013) developed a mixed-integer linear programming model for the two-stage hybrid flow shop environment with setup times, proposed two heuristics and tested two variations: constrained size of the parallel batches and fixed time slots. They provided combinations of the two performance indices versus operator number, assignment, batch size and time slot. The number of tardy jobs as the objective function was intended for a production volume at full capacity in order to reduce penalty for due dates overrun. Computation experiments suggested balancing the two stages by assigning operators proportionally to the setup time requirements and the machine capacity. This way the plant can be considered as a continuous flow line with a given cycle time and synchronized stages, like in flexible balanced lines, with an always changing mix of handled products (medical kits). A simulated reduction of one operator produced no effect on the number of tardy jobs. The consequent slight increase of the makespan was deemed tolerable compared to the 
relevant economic impact of reducing the operator number. It was also advised to switch operators in order to balance the actual setup speed among stages, based on the production plan. The operator assignment was a dominating parameter for the system performance making the examined case more a plant setup and layout design than a scheduling problem.

Rossi, Pandolfi and Lanzetta (2014) extended to $S$ stages $(S \geq 2)$ the hybrid flow shop environment with related parallel batching machines per stage and parallel batches and sequence-independent setup times in order to minimize the number of tardy jobs and the makespan. They proposed various heuristics with their variations based on the critical ratio (CR), a priority rule, which involves the setup times of jobs and the rolling time window for application to the batch scheduling problem. The priority of jobs was determined dynamically at sliding time windows by evaluating the ratio of allowance processing and setup times in the scheduling horizon and two dynamic heuristics, which update the schedule after new jobs enter the system. The authors found an optimal time window, which is about $1 / 4$ compared to Rossi, Puppato and Lanzetta (2013). By such low time allowed for setup, machines start operation more frequently and consequently with smaller batches. For this reason, there was no need to test the system with an additional parameter to limit the machine capacity. As a consequence of the increased batch fragmentation by the lower time window (machine loaded with fewer jobs and starting more frequently), the system performance was improved: system runs found no tardy jobs and lower makespan in all benchmarks considered. However, setup times were fixed in advance and actual heuristics performance may be affected by uncertain times. Besides, as the operator assignment remains the dominating parameter, uncertain times of manual operations strongly affect the plan setup and layout design, which represent the main modules of the examined system. 


\section{Literature on quality improvement}

A quality improvement approach was followed in this work, although the main purpose of the paper is not quality improvement research. The focus on operator modeling is the result of a preliminary system analysis with the purpose of hospital quality improvement. Framing the operator efficiency issue within the system improvement process represents input for quality management and organization modeling research.

Among available quality improvement principles is lean healthcare, approached in Henrique et al. (2016) by value stream mapping (VSM); Krueger, Mellat Parast and Adams (2014) implemented six sigma in a manufacturing plant using qualitative case study methodology. Results include a coding paradigm for the implementation process and implications for managers and practitioners. Stecke and Raman (1995) proposed a model outlining the different sources of flexibility and challenges for plant optimization. Piercy, Phillip and Lewis (2012) showed that staff, management and political resistance to change is a major success limitation in public organizations, consequently among quality management principles outlined, in this work, continual improvement according to ISO 9004 has been followed. Service delivery and materials management with reference to the organization structure was presented by Chenhall (2003) and by Dale, Van Der Wiele and Van Iwaarden (2013).

\section{Problem formulation}

Formally, there is a set of kits (or jobs) $i=1,2, \ldots, N$, for processing in batches on a two-stage flow line with $m_{j}$ related parallel machines included at stage $j$. Each machine $h$ at stage $j$ has a capacity $u_{j}$, a processing time $p_{j}$, a setup speed $v_{j}$ and a batch size $b_{j}$ identical for all the parallel machines of the stage that is a fixed percentage of the machine capacity $\left(\delta \cdot u_{j}\right)$,

$\delta \in[0,1]$. For simplicity the parameter $\delta$ has been neglected [ $\delta=1]$. Each kit $i$ is available from 
a release date $r_{i}$ onwards, has a setup time $s_{i j}$ on a machine of stage $j$, a size $z_{i j}$ expressed with reference to the unit of measurement of the machine capacity at stage $j$ (e.g. number of trays for stage 1 and number of containers for stage 2), a priority $P_{i}$, and a due date $d_{i}$ requested by the operating wards.

For each delivery time window of time $\Delta_{w}$ per day, $w=1, . ., W$, each operator $k=1, \ldots, v_{1}$ at stage 1 and $k=v_{l}+1, . ., V$, at stage 2, available at time $a_{k} \in \Delta_{w}$, carries out with expected efficiency $E_{k}$, setup operations of batch $b=1, \ldots, B$, to be processed by the (first) parallel machine currently available at stage $j$. The setup time $C_{j h b}$ of a parallel batch $b$ to be carried out on machine $h$ at each stage $j$ depends on the number of operators $v_{j}$ (i.e. $v_{1}$ and $V-v_{1}$ ). In the case of a single operator, it is the sum of the setup times of the jobs in the batch.

The completion time of a batch $b$ to be carried out on machine $h$ at each stage $j$ coincides with the completion time of the kits which belong to it, which are all equal to $\left(C_{j h b}+p_{j}\right)$.

The objective is to evaluate $E_{k}$ as a combination of various components detailed below $E_{k} s_{i j}$, which are directly linked to both the completion $C_{i j}$ of job $i$ and the lateness $L_{i}$ of job $i$ for each stage $j\left(L_{i}=C_{i j}-d_{i}\right)$.

By the expected efficiency $E_{k}, k=\left\{1,2, . ., v_{j}\right\}$, stage $j$ can carry out $v_{j}$ units of setup in one time unit. $\left[E_{k} s_{i j} / v_{j}\right]$ is the actual setup time of job $i$ at stage $j$ performed by operator $k$. For line balancing purposes, according to past work, the speeds in the different stages of the sterilization plant should be constant.

The problem is subject to the following constraints

- $\quad$ each job $i$ can be processed by at most one machine for each stage;

- no jobs have agreeable release and due dates (jobs with earlier release date do not necessarily have and earlier due date);

- jobs routing are unidirectional but not identical because few jobs cannot be machinewashed (deleted job); 
- each job size is lower than the machine capacity and many jobs can be batched together respecting the machine capacity constraint;

- $\quad$ each job must be processed within $D$ minutes from the release date (no-wait condition on stage 1);

- no preemption is allowed (operations will be uninterrupted);

- if the priority of job $i$ is higher than the priority of job $l$, then job $i$ must be completed before job $l$.

\section{The proposed model}

To consider the variability on the duration of manual operations and their causes (skill, stress, motivation etc.) we propose an operator model as a combination of different efficiency factors and explore its effect on modeling the main modules of a general production system as in Figure 1.

The expected setup time $s_{i j}$ can be described as a continuous random variable with a given probability density function to be estimated by sampling. Standard times can be evaluated by systematically or randomly measuring the operation time, by different operators at different times of different shifts. The distribution type should also be assessed.

Let $\xi_{i j o}$ be the $o^{\text {th }}$ time measured of job $i$ among $N$ jobs on stage $j$ of $S$ stages. The following mean of $O$ observations is the standard time $s_{i j}$ for job $i$ on stage $j$ :

$s_{i j}=\frac{1}{O} \sum_{o=1}^{O} \xi_{i j o}, \quad i=1, . ., N, \quad j=1, . ., S$

Standard times $s_{i j}$ are an estimate on a statistical basis and are influenced by different factors, which affect the performance producing an actual activity time $\sigma_{i j}$ 


$$
\sigma_{i j}=E_{k} s_{i j}
$$

The performance degradation is the reciprocal of the efficiency:

$$
E_{k}=1 / \mathrm{K}_{k}
$$

$E_{k}$ may vary with time.

The average performance corresponds to $E_{k}=1$. When $E_{k}$ is included in the range $(0,1], \mathrm{K}_{k} \geq$ 1 ; shorter activity time means better efficiency (lower $E_{k}$ ). The effect of the performance degradation on the resulting $\mathrm{K}_{k}$ is to increase the standard time by $1 / \mathrm{K}_{k}$ increasing $s_{i j}$ up to $s_{i j} / \mathrm{K}_{k}$.

$\mathrm{K}_{k}$ can be expressed as the algebraic sum of various $\mathrm{K}_{x}$ coefficients and/or the product of $\mathrm{K}_{y}$ coefficients

$\mathrm{K}_{k}=\sum_{x} \mathbf{K}_{x}+\prod_{y} \mathbf{K}_{y}$

to show the effect of various contributions to performance. Although a more complex formulation is possible, equation (4) seems suitable to cover the generality of real cases.

Each factor affects performance degradation/improvement, being lower/greater than 1 . The value of 1 represents a negligible effect on the average operator performance. 0 means totally inefficient or absent operator. Coefficient values exceeding 1 represent a better operator or performance. The negative case $(-\propto, 0)$, corresponding to the presence of an operator degrading the performance of one or more other operators (nagging operator), can also be considered.

In addition to qualitative observations, the significance of each coefficient on the overall 
performance can also be statistically assessed a posteriori.

Possible uses of the proposed time standardization model include system simulation, capacity analysis, operator assignment, layout design, scheduling, performance assessment and continual improvement (Dallery and Gershwin 1992) as shown in the following application, which focuses on the shaded areas of the System Run modules in Figure 1. Furthermore, the expected efficiency parameter completes the MILP formulation of the system design in Figure 1 and the related scheduling problem.

Notation

$j \quad$ stage index $, j=1, \ldots, S$, where $S$ is the total number of stages

$h \quad$ machine index, $h=1, \ldots, M_{j}$, where $M_{j}$ is the total number of machines at stage $j$

$i \quad$ job index, $i=1, \ldots, N$, where $N$ is the total number of jobs to be processed

$b \quad$ batch index $, b=1, \ldots, B$, where $B$ is the total number of batches

$k \quad$ operator index, $k=1, . ., v_{l}, v_{l}+1, . ., V$, where $V$ is the total number of operators and $v_{l}$ is the number of operators at stage 1 (also representing the setup speed at stage 1)

$w \quad$ delivery time window index, $w=1, . ., W$, where $W$ is the number of time windows per day

$\Delta_{w} \quad$ duration of delivery time window $w, w=1, \ldots, W$ in minutes

$r_{i} \quad$ release date of job $i$; coincides with the beginning of delivery time windows

$d_{i} \quad$ due date of job $i$

$m_{j} \quad$ number of parallel machines at stage $j$

$p_{j} \quad$ processing time of parallel machines at stage $j$ 
$u_{j} \quad$ machine capacity at stage $j$ (i.e. all the machines at stage $j$ have the same capacity, but the unit of measurement can change at each stage if a job is split in medical devices, trays, containers etc.)

$z_{i j} \quad$ size of job $i$ at stage $j$ expressed with the to unit of measurement of the machine capacity

$P_{i} \quad$ priority of job $i$ at stage 1

$s_{i j} \quad$ setup time of job $i$ at stage $j$, calculated according to (1)

$v_{j} \quad$ units of setup carried out in one time unit

$a_{k} \quad$ release date of operator $k$

$E_{k} \quad$ efficiency of operator $k$

$C_{i j} \quad$ completion time of job $i$ at stage $j$

$C_{j h b} \quad$ completion time of batch $b$ of machine $h$ at stage $j$

$L_{i} \quad$ lateness of job $i$, at the last stage $S, L_{i}=C_{i S}-d_{i}$.

$U_{i} \quad$ completion status of job $i$ represented by $U_{i}=1$ if $L_{i}>0,0$ otherwise

BigM a large number $\rightarrow+\infty$

Decision variables

$X_{i j h} \quad 1$, if job $i$ is assigned to machine $h$ at stage $j$

0 , otherwise

$Y_{k j} \quad 1$, if operator $k$ is assigned to stage $j$

0 , otherwise

$Z_{i j h b} \quad 1$, if job $i$ is assigned to batch $b$ of machine $h$ at stage $j$

0 , otherwise 


\section{The mixed integer linear programming model}

A mixed integer problem formulation allows the formalization of the system modules. As detailed below, the MILP includes (but is not limited to) the operator efficiency, which represents the proposed solution to deal with uncertain times in the job shop scheduling problem.

Example objective functions for current case are:

- minimizing the total number of operators, organization criterion $\min V$

- minimizing the completion time, process efficiency criterion $\min \mathrm{C}_{\max }$

- minimizing the number of tardy jobs, service level criterion $\min \sum U_{i}$

- minimizing the maximum job lateness, just in time criterion

$$
\min \max L_{i}
$$

Variations include, weighted tardy jobs, maximum lateness, machine/operator workload and other weighted combinations of various objective functions.

Minimal process constraints:

$$
\begin{aligned}
& \sum_{h=1}^{M_{j}} X_{i j h}=1 \\
& i=1, . ., N \\
& j=1, . ., S
\end{aligned}
$$




$$
\begin{aligned}
& \sum_{j=1}^{S} \sum_{b=1}^{B} Z_{i j h b}=1 \\
& i=1, . ., N \\
& h=1, . ., M_{j} \\
& \sum_{i=1}^{N} z_{i} \cdot Z_{i j h b} \leq u_{j} \\
& h=1, . ., M_{j}, j=1, . ., S \\
& b=1, . ., B \\
& \sum_{j=1}^{S} \sum_{k=1}^{V} Y_{k j}=v_{1}+\sum_{j=2}^{S} \sum_{k=v_{1}+1}^{V} Y_{k j}=V \\
& j=1, . ., S \\
& k=1, . ., v_{1}, v_{1}+1, . ., V \\
& C_{i j} \geq C_{j h b}-\operatorname{BigM}\left(1-Z_{i j h b}\right) \\
& h=1, . ., M_{j}, j=1, . ., S \\
& i=1, . ., N, b=1, . ., B \\
& C_{j h b} \geq C_{i(j-1)}+\left(E_{k} \cdot s_{i j}\right)+p_{j}-\operatorname{BigM}\left(1-Z_{i j h b}\right) \\
& h=1, . ., M_{j}, j=2, . ., S \\
& i=1, . ., N, k=1, . ., V \\
& b=1, . ., B \\
& C_{j h b} \geq \max \left\{C_{i(j-1)}+\left(E_{k} \cdot s_{i j}\right), C_{j h(b-1)}\right\}+p_{j}-\operatorname{BigM}\left(1-Z_{i j h b}\right) \quad h=1, . ., M_{j}, j=2, . ., S \\
& i=1, . ., N, k=1, . ., V \\
& b=2, . ., B \\
& C_{i j} \geq \max \left\{C_{i(j-1)}+\left(E_{k} \cdot s_{i j}\right), C_{o j}\right\}+p_{j}-\operatorname{BigM}\left(2-Z_{i j h b}-Z_{o j h(b-1} h=1, . ., M_{j}, j=2, . ., S\right. \\
& i=1, . ., N, k=1, . ., V \\
& b=2, . ., B \\
& \left.C_{i(j+1)} \cdot Z_{i j h b} \geq \min _{i=1, \ldots, N}\left[C_{i j} \cdot Z_{i j h b}\right]+\left[\frac{\sum_{i=1}^{N}\left(E_{k} \cdot s_{i(j+1)}\right) \cdot Z_{i j h b}}{v_{j}}\right]+\begin{array}{r}
h=1, . ., M_{j}, j=1, . ., S-1 \\
p_{j}-\begin{array}{c}
i, . ., N, k=1, . ., V \\
b=1, . ., B
\end{array}
\end{array}\right] \\
& -\operatorname{BigM}\left(1-Z_{i j h b}\right) \\
& C_{i 1}-p_{1} \leq r_{i}+D \\
& i=1, . ., N \\
& w=1, . ., W
\end{aligned}
$$

Constraint (9) assures that each job is only assigned to one machine for each stage. Constraint (10) guarantees that each job is assigned exactly to one batch for each stage. Constraint (11) assures that the number of jobs included in a parallel batch does not exceed the capacity of the 
assigned machine. Constraint (12) describes the relationship between setup speed at each stage and number of operators.

Constraint (13) assures that the completion time of a job cannot be lower than the completion time of the belonging batch. Constraint (14) describes the relation between job completion time and completion time of the belonging batch at the subsequent stage. Similarly to (14), the relation in constraint (15) is between two subsequent batches of the same machine. Again in constraint (16) the relation is between two jobs of subsequent batches on the same machine. In constraint (17) the job completion time is higher than the early completion time at the previous stage plus the sum of the activity time and the actual batch setup time defined as

$$
\left[\left(\sum_{i=1}^{N}\left(E_{k} \cdot s_{i j}\right) \cdot Z_{i j h b}\right) / v_{j}\right], \quad j=1, \ldots, S, \quad k=1, . ., V
$$

Finally, the no-wait constraint (18) indicates each job must be processed at the stage 1 within a fixed due date $D$ from its release date $r_{i}$.

Examples of system modules are plant setup, machine capacity and line balancing. Equations (14) to (17) are the expression of a hybrid direct observation/synthetic method for activity time estimation.

\section{Plant setup}

To maximize the plant efficiency, the operators number should be minimized according to the objective function in equation (5). The total operator number per shift and their assignment can be considered as a layout design problem.

The assignment of operators to the two stages of the MILP by the parameter $v_{1}$ is a degree of freedom and their total number $V$ is fixed from the capacity analysis. 


\section{Machines capacity}

For the machine capacity constraint (11) in the MILP, the following expression can be used

$$
\gamma_{w}=\frac{M_{j} u_{j}}{\sum_{i \in \Delta_{w}} z_{i j}} \quad j=1,2 \text { and } w=1, . ., W
$$

where $M_{j} u_{j}$ is the machine capacity at stage $j$ required to achieve full capacity $\left[\gamma_{w} \geq 1\right]$ and $i$ is the number of jobs delivered at the beginning of delivery time windows $\Delta_{w}$.

\section{Line balancing}

The operators assignment can be based on line balancing criteria, using expression

$$
\min \left[\left(\frac{1}{v_{1}} \sum_{k=1}^{v_{1}} \sum_{i=1}^{N}\left(E_{k} \cdot s_{i 1}\right)-p_{1}\right)-\left(\frac{1}{v_{2}} \sum_{k=v_{1}+1}^{V} \sum_{i=1}^{N}\left(E_{k} \cdot s_{i 2}\right)-p_{2}\right)\right]
$$

which requires the knowledge about the number of jobs flowing at each stage.

\section{Application}

This section of the paper outlines the use of standardized activity times according to the model in Figure 1, in some of the main system modules, such as plant setup, capacity analysis, operator assignment and scheduling.

The application field is health services, the sterilization plant of one of the largest European hospitals, Azienda Ospedaliero-Universitaria Pisana (AOUP) of Pisa, servicing the center of Italy. From the merging of two existing hospitals, it hosts about 1,600 beds, 900 recoveries 
per day and 2,500 specialist exams.

Hospital sterilization aims at eliminating infectious risks coming from the reuse of medical devices in surgical operations (ISO 17665). Reusable medical devices (RMDs) can be defined as instruments used in clinics, surgeries or laboratories that can be reused. After use in operating units, medical devices are sent to the Central Sterile Services Department (CSSD).

This study has been requested by the hospital top management with the purpose of improving the plant efficiency by increasing effectiveness, reducing costs or both, while still satisfying the tight constraints and the high standards required in public health services.

Increasing the efficiency of sterilization services, e.g. the plant throughput or the number of medical devices sterilized per day and per operator, increases the medical device rotation between operations and helps keep stocks low and cut purchasing costs. Inefficiencies, like a delayed delivery of medical devices, causes surgery to be rescheduled with heavy not only economical consequences, in the order of one thousand Euros per hour, including health, hospital image, legal implications etc.

At AOUP some 17,000 reusable medical devices (RMDs) are available, belonging to 800 medical kits, coming from 144 operating wards in 15 hospital departments, including physical examinations, operating theaters (surgical kits), chemical labs, etc. Medical devices are enclosed inside one or more containers of three standard sizes $(600 \times 300 \times 210,300 \times 300 \times 210$ and $120 \times 300 \times 50$ expressed in millimeters) to form kits; kits consist of 1 to 9 containers, each containing between 1 and 120 medical devices, like scalpels, knives, scissors etc. depending on the specialty and surgery type. More than 1,500 medical devices processed per day involving manual operations make this a large-scale case. An average of 110 surgical kits from 54 operating theatres is processed per day, with tight due date tolerances and a scheduling horizon of few days. Some of the kits are classified as life saving and have higher 
priority.

\section{Process description}

The use cycle of medical devices is summarized in Figure 2. After use in operating wards (e.g. surgery), medical devices undergo pre-disinfection (not shown). Kits are delivered from operating wards to the sterilization central at fixed time windows.

Kits achieve a standardized sterility assurance level by (i) machine-washing and (ii) steam sterilization. Because of the physical separation between the two areas, the assignment of operators is constant throughout the duration of each 8 hours shift. The sterilization plant is operational 24 hours a day. The total number of operators and their assignment to each of the two areas is an example of plant setup.

The sterilization plant has two machine stages including respectively, six washer disinfectors and five steam sterilizers. These two stages with machine operations are preceded and followed by manual operations. After setup time, each kit is processed by one of the parallel machines at each stage. The machine time on both stages of the sterilization plant is the same (70 minutes) for line balancing purposes.

Stage (i)

In the rinsing area, containers are emptied into basins. Medical devices are washed manually and placed into the metal trays of washer disinfectors, which have two different sizes 240 and $480 \times 250 \times 50$ millimeters. Rinsing and mechanical washing prevents residual dirt to create a sort of barrier to microorganisms (particularly spores) and prevent their destruction and invalidating the whole process. Washing needs to start within one hour of receiving a kit for sanitary reasons (to reduce drying of organic material). 
Trays are inserted into the carts of washer disinfectors, which have five levels. If one or more medical devices are higher than the pitch of two consecutive levels one level can be taken off. Wash carts are filled up to the machine capacity, which is the same for all machines.

\section{Stage (ii)}

After the disinfector cycle, trays are taken out into the prep \& pack area, where medical devices are inspected for cleaning, integrity and completeness, dried and placed into containers. Drying is not only to avoid corrosion, but also to allow proper exposure of the material to the sterilizing agent. Containers are placed on the two levels of the loading platforms of the steam sterilizers, up to the capacity of 48 small, 24 medium or 12 large containers.

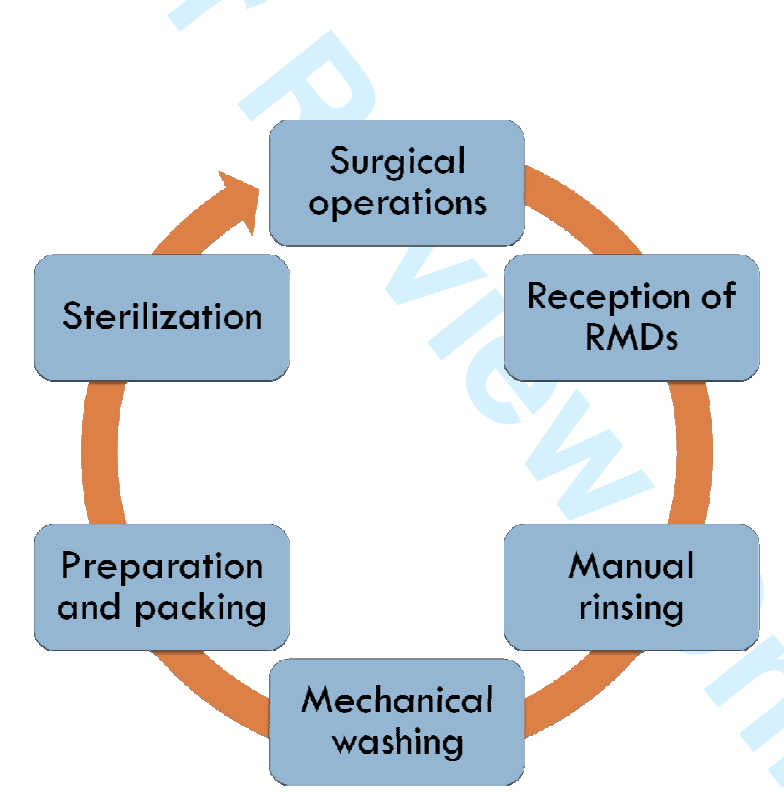

Figure 2 Overview of the sterilization process.

\section{Problem solving approach}

This section outlines the potential process inefficiencies. 
Figure 3 shows the relationship among the main actors, sterilization plant, operating wards and top management, and the material and information flow, between the first two, which is governed by the last one.

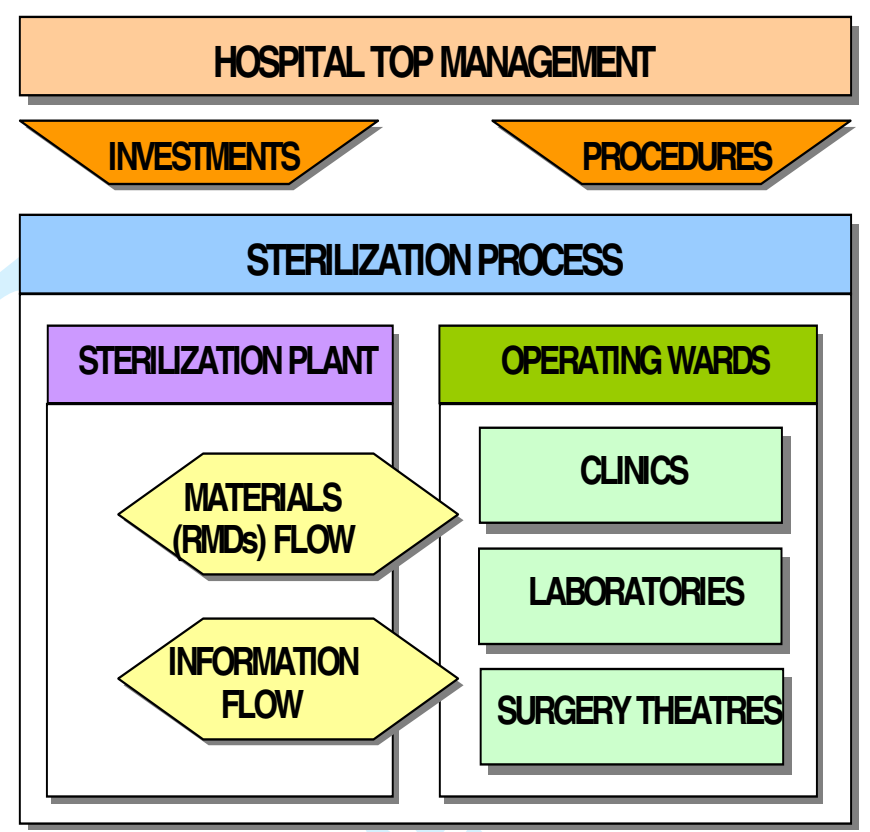

Figure 3 The main material and immaterial flows involved in the sterilization process, which integrates and impacts most hospital operating wards. Improvement is driven by the top management.

This study being supported by the top management of the hospital, improvement actions involve not only the sterilization plant level, but also the overall system (hospital), because of the bi-directional material and information flow with external operating wards shown in Figure 3. Instead of redesigning the whole sterilization process (radical improvement), an incremental improvement approach has been conservatively selected, from a sterilization plant perspective. Among the reasons for an incremental approach are the initially declared 
constraints: natural resistance to change, tight health and staff norms and regulations, keeping balanced status quo powers and equilibriums, labor unions oppositions.

Modeling the operator efficiency in order to cope with time uncertainty has been selected because of its expected faster and higher returns and system performance increase, producing a minimal impact.

From a preliminary analysis carried out by observations on the field and interviews, the main causes of inefficiencies of the sterilization process have been summarized by the Ishikawa's cause and effects diagram in Figure 4.

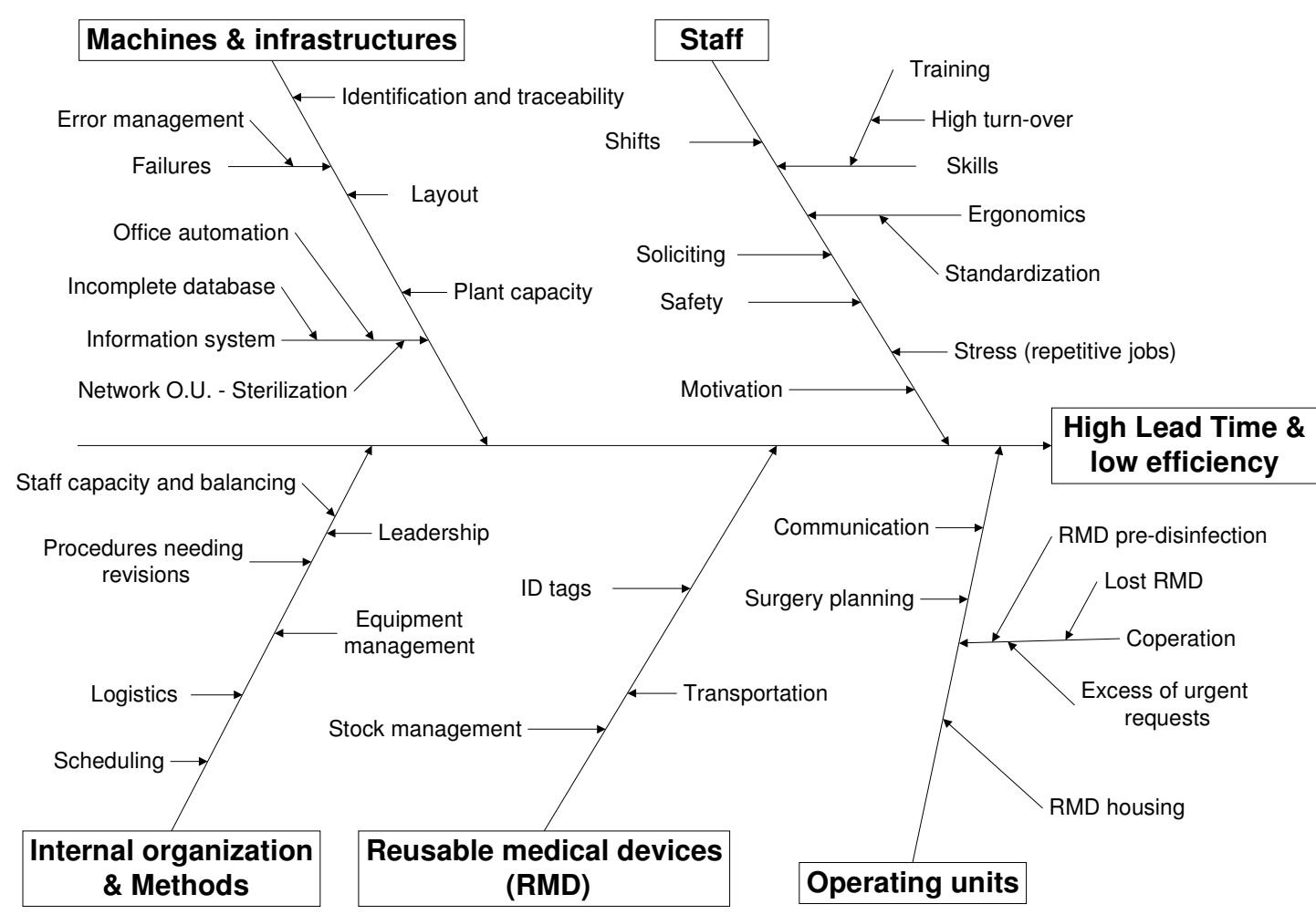

Figure 4 Overview of the main improvement areas of the sterilization plant at the examined AOUP hospital.

Causes have been identified primarily considering the 4 Ms: manpower = staff; machines - 
including infrastructures; materials = medical devices; methods/organization. An internal viewpoint, involving organization, staff management and machines, and an external viewpoint, concerning order management, stock management, information system, has also been considered in order to include a fifth element (operating units) to explicitly show the internal/external interconnections between the sterilization unit and the operating units.

As shown in Figure 3, improvement options can also be classified in two groups: investments in infrastructure (e.g. information system and layout redesign) and reorganization (e.g. business process reengineering, approach by processes, new procedures and protocols, staff management). Problems can certainly be solved by investments, but this option is not always sustainable. Increase of system resources (more operators, more machines and more medical devices) would only increase effectiveness (medical devices availability), not necessarily affecting efficiency, as opposed to lean production principles, and has not been considered.

In this work, the sterilization plant has been monitored for several months to collect information and quantitative data, which have been analyzed in order to provide returns, by minimizing investments and the impact of changes. The sterilization process has been approached in analogy with manufacturing practice, following the steps outlined in Figure 1.

\section{Process planning}

Regarding the need for standardization pointed out in Figure 4 - staff, in analogy with the manufacturing practice, current sterilization process has been described by the process plan in Table 1. 
Table 1 Example of process plan for medical kit.

\begin{tabular}{|c|c|c|c|}
\hline & & \multicolumn{2}{|l|}{$\begin{array}{l}\text { STERILIZATION PROCESS PLAN } \\
\text { AOUP hospital }\end{array}$} \\
\hline $\begin{array}{l}\text { Kit name: } \\
\text { Thyroid }\end{array}$ & & Ward: General surgery & $\begin{array}{l}\text { Sheet } \\
1 \text { of } 1\end{array}$ \\
\hline $\begin{array}{l}\text { Updated: } \\
\text { 21/09/10 }\end{array}$ & & Priority: $\square$ normal $-\square$ life & \\
\hline \multirow[t]{2}{*}{ Stage $j$} & Phase & Subphase & $\begin{array}{l}\text { Time } \\
{\left[\text { min.] } s_{i j}\right.}\end{array}$ \\
\hline & 10 & 10.1 - kits check-in & \\
\hline $\begin{array}{l}\text { Setup } \\
\text { (washing) }\end{array}$ & 20 & $\begin{array}{l}20.2 \text { - manual rinsing } \\
20.3 \text { - medical devices loading on trays } \\
20.4 \text { - tray loading on wash cart } \\
20.5 \text { - cart loading on washing machine to } \\
\text { specified machine capacity (batch } \\
\text { forming and close) }\end{array}$ & 4.9 \\
\hline 1 & 30 & 30.1 - mechanical washing & 70 \\
\hline $\begin{array}{l}\text { Setup } \\
\text { (prep \& } \\
\text { pack) }\end{array}$ & 40 & $\begin{array}{l}40.1 \text { - cart and tray unloading } \\
40.2 \text { - medical devices visual inspection } \\
40.3 \text { - medical devices preparation } \\
40.4 \text { - list check } \\
40.5 \text { - packing into containers } \\
40.6 \text { - containers loading into autoclave to } \\
\text { specified machine capacity (batch } \\
\text { forming and close) }\end{array}$ & 11.5 \\
\hline \multirow[t]{2}{*}{2} & 50 & $50.1-$ steam sterilization & 70 \\
\hline & 60 & $\begin{array}{l}60.1 \text { - containers unloading } \\
60.2 \text { - kits forming } \\
60.3 \text { - shipping }\end{array}$ & \\
\hline
\end{tabular}

Each of the several hundreds of kits has its own process plan, which can be described by a document like the one in Table 1.

It can be noticed that for current plant, process plans are similar for all kits (same routing) and the line layout is of the flow shop type. Machine time on the two stages is the same for all kits. As in past work, manual operations are considered as machine setup, with time $s_{i j}$. Only manual operations times $s_{i j}$ differ from kit to kit, so plant capacity, throughput, and scheduling is essentially affected by the mix of kits, by their respective times. The setup time in the first stage is always lower than that in the second stage (as shown in Figure 5): in the first, kits are disassembled into individual medical devices, which are checked and reassembled into containers in the second. 
The manual setup times $E_{k} s_{i j}$ for different kits and operators have been determined by direct observation and corrected by the efficiency parameters of the individual operator performance as detailed below.

\section{Operator efficiency}

According to our synthetic method for activity time estimation, standard times have been evaluated by randomly measuring the operation time for different medical kits on the two stages, by different operators at different times of different shifts. The measured activity times have shown a normal distribution with high confidence $(\mathrm{P}$-value $=0.05)$.

Based on observations and interviews, the most relevant factors in equation (4) for current case have been isolated and quantified and result in the product $\mathrm{K}_{k}$ from of the following coefficients:

$\mathrm{K}_{k}=\eta_{k} \mathrm{~K}_{p} \mathrm{~K}_{d}-\mathrm{K}_{f}$

The negative case $(-\propto, 0)$, corresponding to the presence of a nagging operator degrading the performance of one or more other operators, has not been contemplated.

The main factors, which have been isolated and quantified, are:

- an operator specific coefficient $\eta_{k}$, depending on skill, motivation and training; $\eta_{k}=1$ for the average operator;

- work condition (environmental noise, repetitive work, use of force, environmental temperature and light, affecting efficiency), which causes a progressive reduction of efficiency towards the end of each shift.

Work conditions influence the operator fatigue and consequently its efficiency. The most relevant aspects in current case are: 
- standing, environmental noise and repetitive work, which causes a progressive reduction of efficiency towards the end of each shift and is estimated as a linear coefficient, $\mathrm{K}_{f}=0.0005 t ; t$ is the time in minutes elapsed from the beginning of each shift and producing a $24 \%$ performance reduction at the end of a 8 hour shift;

- use of force, environmental temperature and light, are approximately constant and do not affect operators time.

The actual availability of operators needs to be corrected to take into account also the following aspects:

- personal needs, which are always present in different proportions, like restroom, phone calls, coffee breaks, and have been estimated as 5 minutes per hour or $91.67 \%$ of the shift time; $\mathrm{K}_{p}=0.9167$;

- delays, essentially due to system inefficiency, which cause interruption of work, like urgent requests, soliciting, missing medical devices or kit information and have been estimated as 15 minutes per 8 hour shift or $96.88 \% ; \mathrm{K}_{d}=0.9688$.

\section{Standard times}

According to the direct observation method for activity time estimation, standard times for each kit $s_{i j}$ for the two phases have been determined as the mean of all operators. Among all operators, whose performance are normally distributed according to Table 2 and Table 3, some are more efficient and some are less efficient according to the parameter $\eta_{k}$, which can be estimated as

$$
\left(1-t_{\alpha / 2} \cdot \frac{s}{\sqrt{O}}\right)<\eta_{k}<\left(1+t_{\alpha / 2} \cdot \frac{s}{\sqrt{O}}\right)
$$


where $t_{\alpha / 2}$ is the Student's variable for a significance level $\alpha$ and $s$ is the standard deviation of a sample of $O$ observations of $\eta_{k} / s_{i j}$ on all operators; 11 is the average number of active operators in a shift, ranging from 9 to 15 , taken from a total of 21 available. Current laws do not allow assessing the individual operator performance for a public hospital in Italy. In general, a more detailed estimation of the individual operators' efficiency would allow more accurate system performance analyses (capacity, productivity etc.), so $\eta_{k}$ has been included in the current model.

Only the component $\eta_{k}$ of the operator performance degradation $K_{k}$ in (22) is specific for operator $k$.

The standard times for 22 kits in the two stages are listed in Figure 5. The standard times can also be represented with a normal distribution with respectively mean 3 and 7 and standard deviation of 6 and 12 on the two stages. These data expressed in minutes and upper limited at two standard deviations have shown to include $70 \%$ of all possible values and allow generating random samples of kits to be processed by the sterilization plant on a given time period to simulate the system performance. 


\section{Plant setup}

In this application, the operator assignment has been determined considering peak day data. In the next paragraph the capacity analysis is also based on historical kit requests.

By the relationship between setup speed at each stage and number of operators, included in constraint (12), and the no-wait constraint (18), which indicates each job must be processed at stage 1 within a fixed due date ( $D=60$ minutes at AOUP hospital), the plant capacity should be proportional to the setup speed described by (19).

\section{Plant capacity}

Each 24 hours work day is divided into 11 time windows for kits reception of different duration $\Delta_{W}\left(\Delta_{1}, \Delta_{2}, \ldots, \Delta_{11}\right)$ listed in Table 6 in Appendix. 
An estimation of the plant capacity $\lambda_{w}$ at the first stage (manual rinsing) is given by

$$
\lambda_{w}=\frac{v_{1}^{*} \cdot \Delta_{w}}{\sum_{i}\left(E_{k} \cdot s_{i 1}\right)}
$$

where $v_{1}^{*}$ is the number of operators at stage $j=1$ required to achieve full capacity $\left[\lambda_{w} \geq 1\right], i$ is the number of kits delivered at the beginning of the delivery time window $\Delta_{w}$, as shown in Figure 6, and the rate $\sum_{i}\left(E_{k} \cdot s_{i 1}\right) / v_{1}^{*}$ is achieved by constraint (17) where the jobs completion time for stage 1 is higher than the sum of setup times (parallel batch).
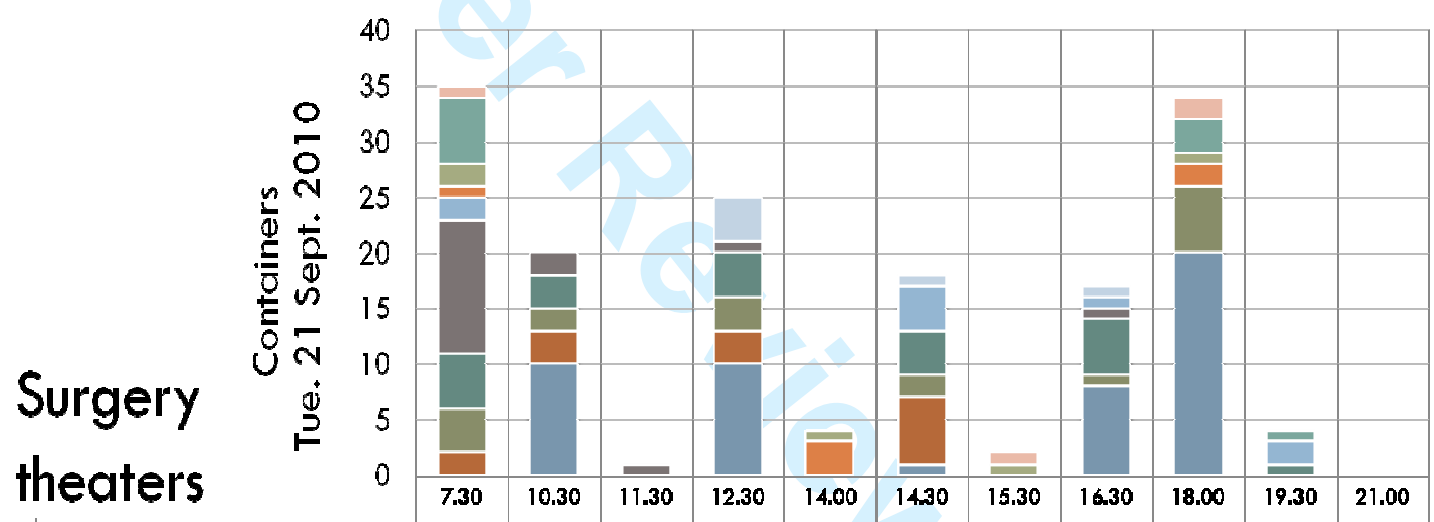

\begin{tabular}{|c|c|c|c|c|c|c|c|c|c|c|c|}
\hline CHIRURGIAGENGRALE $4^{\circ}$ - 97 & 0 & 0 & 0 & 4 & 0 & 1 & 0 & 1 & 0 & 0 & 0 \\
\hline CHIRURGIASENOLOGICA - 48 & 0 & 0 & 0 & 0 & 0 & 0 & 0 & 0 & 0 & 0 & 0 \\
\hline CHIRURGIA VASCOLARE - 43 & 1 & 0 & 0 & 0 & 0 & 0 & 1 & 0 & 2 & 0 & 0 \\
\hline CHIRURGIA GENERALE - 42 & 6 & 0 & 0 & 0 & 0 & 0 & 0 & 0 & 3 & 1 & 0 \\
\hline CHIRURGIATORACICA - 02 & 2 & 0 & 0 & 0 & 1 & 0 & 1 & 0 & 1 & 0 & 0 \\
\hline CARDIOCHIRURG|A-01 & 1 & 0 & 0 & 0 & 3 & 0 & 0 & 0 & 2 & 0 & 0 \\
\hline UROLOGIA UNIVERSITARIA - 125 & 2 & $\mathbf{0}$ & 0 & 0 & 0 & 4 & 0 & 1 & 0 & 2 & 0 \\
\hline - UROLOGIA OSPEDALIRA - 109 & 12 & 2 & 1 & 1 & 0 & 0 & 0 & 1 & 0 & 0 & 0 \\
\hline CHIRURGIA GEN. $2^{\circ}-124$ & 5 & 3 & 0 & 4 & 0 & 4 & 0 & 5 & 0 & 1 & 0 \\
\hline - CHIRURGIAGEN $4^{\circ}-124$ & 0 & 0 & 0 & 0 & 0 & 0 & 0 & 0 & 0 & 0 & 0 \\
\hline OTORINO - 101 & 4 & 2 & 0 & 3 & 0 & 2 & 0 & 1 & 6 & 0 & 0 \\
\hline -CHIRURGIAGEN, COLON RETTALE - 107 & 2 & 3 & 0 & 3 & 0 & 6 & 0 & 0 & 0 & 0 & 0 \\
\hline DCULISTICA - 106 & 0 & 10 & 0 & 10 & 0 & 1 & 0 & 8 & 20 & 0 & 0 \\
\hline
\end{tabular}

Figure 6 Kits per surgery theatre and time window on a peak date. 
$E_{k}$ is operator dependent by the $\eta_{k}$ parameter in (22), and is defined at the scheduling phase when the kit is assigned to the actual operator. In this application, $E_{k}$ is independent from the single operator and it is used to estimate the efficiency of a manual operation depending on the features of a medical kit, particularly size, dirt level and handling difficulty.

Similarly, expression (24) can be used to optimize the shift timing and the delivery time windows, based on historical data on kit requests and delivery.

A requirement is that all kits received at the plant be processed within one hour from arrival, by the no-wait constraint at stage 1 (18). From monitoring the plant for several months, it has been shown that Tuesdays and Wednesdays are peak days. Figure 6 lists the kit arrivals used in the capacity estimation considering peak day data.

A similar expression has been derived to determine the number of operators on the second stage in order to achieve a balanced flow of kits. It has been supposed that the same amount of kits is delivered at the second stage after the machine washing time (70 minutes). Based on the number of cycles, time windows $\Lambda_{1}$ to $\Lambda_{5}$ on the second stage have been determined as in Table 7 in Appendix.

\section{Line balancing}

Considering that with current case the machine time $p_{j}$ is constant on the two stages, in a first approximation the operators assignment can be based on balancing the setup speeds on the two stages:

$$
\min \frac{\sum_{i=1}^{N} \sum_{\kappa=1}^{\mathrm{v}_{1}}\left(E_{k} \cdot s_{i 1} / v_{1}\right)}{\sum_{i=1}^{N} \sum_{k=1}^{\mathrm{v}_{2}}\left(E_{k} \cdot s_{i 2} / v_{2}\right)} \quad \mathrm{k}=1, \ldots, V
$$


with the proportion of 1 operator at the first stage versus 2.46 at the latter, based on the kit input in Figure 6 and the setup times in Figure 5.

An alternative estimation of the proportion at the two stages is given by the ratio between the maximum times (mean plus two standard deviations of respectively 15 and 31 minutes) of the standardized distributions hypothesized above, yielding a ratio of 2.07 .

\section{Results}

The actual performance degradation parameter for the average operator $\left(\eta_{k}=1\right)$ as a function of time $t$ in minutes is the product of the main coefficients pointed out in the Application section

$\mathrm{K}_{k}=0.8881-0.0005 t$

The activity times for four kit types from 12 different operators are listed in Table 8 in Appendix. Nurses included in the total number of 21 available operators have not been considered because they are only involved in prep \& pack.

Table 2 and Table 3 list the P-value for different normality tests, which do not require that distribution and parameters be completely specified and known. Strong evidence is shown for the null hypothesis, i.e. all distributions of operator time are normal for the examined case, according to the statistics software R (ver. 2.11.1, last acc. 02/2016 from http://www.rproject.org/). As an example, the Lilliefors test only applies to the procedure of using the Kolmogorov-Smirnov test statistic with estimated null distribution when the null distribution is assumed to be normal but not all parameters are known. This conclusion allows reducing the size of samples to estimate the statistical parameters of interest and to minimize the effort to measure the activity times. The elected sample size is 12 . 
Table 2 Normality test for 12 measurements of washing times on four among the most frequent kits, listed in Table 8 in Appendix (minimum significance level 0.05).

\begin{tabular}{|c|c|c|c|c|}
\hline \multirow{2}{*}{ Normality test / kit name } & Cataract & Thyroid & $\begin{array}{c}\text { Intestinal } \\
\text { surgery }\end{array}$ & Faco ozil \\
\cline { 2 - 5 } & \multicolumn{4}{|c|}{ P-value } \\
\hline Shapiro - Wilk & 0.12 & 0.91 & 0.29 & 0.75 \\
\hline Lilliefors & 0.27 & 0.88 & 0.03 & 0.87 \\
\hline Jarque - Bera & 0.55 & 0.82 & 0.72 & 0.65 \\
\hline Cramer - Von Mises & 0.20 & 0.82 & 0.10 & 0.86 \\
\hline Anderson - Darling & 0.15 & 0.87 & 0.14 & 0.82 \\
\hline
\end{tabular}

Table 3 Normality test for 12 measurements of prep \& pack times on four among the most frequent kits, listed in Table 8 in Appendix (minimum significance level 0.05).

\begin{tabular}{|c|c|c|c|c|}
\hline \multirow{2}{*}{ Normality test / kit name } & Cataract & Thyroid & $\begin{array}{c}\text { Intestinal } \\
\text { surgery }\end{array}$ & Faco ozil \\
\cline { 2 - 5 } & \multicolumn{4}{|c|}{ P-value } \\
\hline Shapiro - Wilk & 0.13 & 0.11 & 0.21 & 0.28 \\
\hline Lilliefors & 0.55 & 0.15 & 0.38 & 0.39 \\
\hline Jarque - Bera & 0.17 & 0.24 & 0.63 & 0.71 \\
\hline Cramer - Von Mises & 0.39 & 0.13 & 0.40 & 0.26 \\
\hline Anderson - Darling & 0.25 & 0.13 & 0.30 \\
\hline
\end{tabular}

The number of kits being in the order of hundreds, in order to minimize the number of observations on the field according to the proposed hybrid direct observation/synthetic method, only a subset of 33 kits has been considered and the following metric has been investigated. 
The processing time for each kit on each of the two manual phases depends on three main parameters:

- the number of devices included in the kit;

- the presence of dirt, like dried organic material;

- the handling difficulty, like the presence of sharp, tiny, difficult to access or delicate devices.

The last two parameters have been determined by asking specialized operators to classify known kits in three classes of increasing dirt level and handling difficulty, preliminarily defined as low, medium and high. An example of classification is shown in Figure 7.

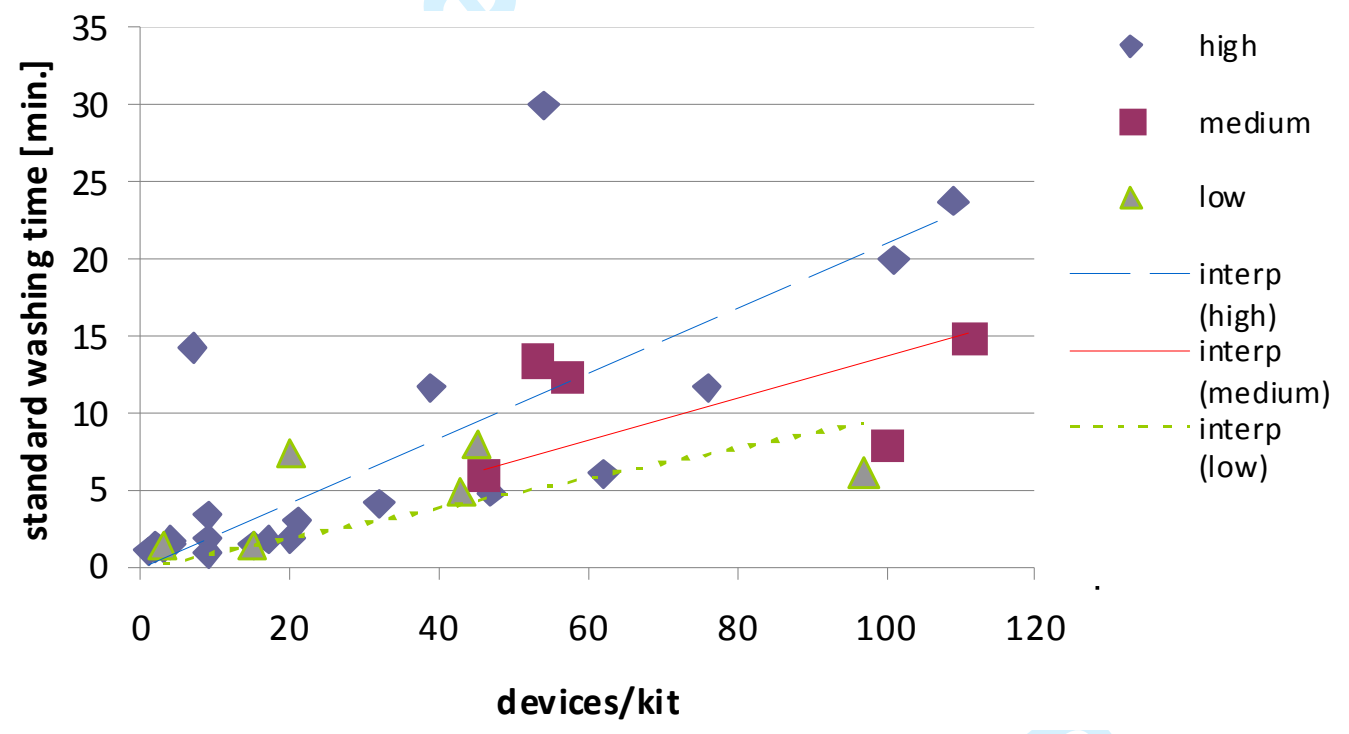

Figure 7 Standard time for the washing operation as a function of the number of devices included in each kit classified in three classes for presence of dirt.

It can be noticed that values are increasingly scattered, that is probably because in general, the variance of a task's time increases with its complexity (Becker and Scholl 2006). Values far from the belonging linear interpolation line are probably caused by subjective assignment of the dirt level, which is a qualitative parameter. 
Imposing an intercept in the origin, the linear coefficients for the three dirt levels (low, medium and high) are respectively $0.097,0.137$ and 0.211 minutes per each device included in a kit. These data show that kits in the medium and high classes take about $40 \%$ and $110 \%$ more time to be rinsed than those in the low class.

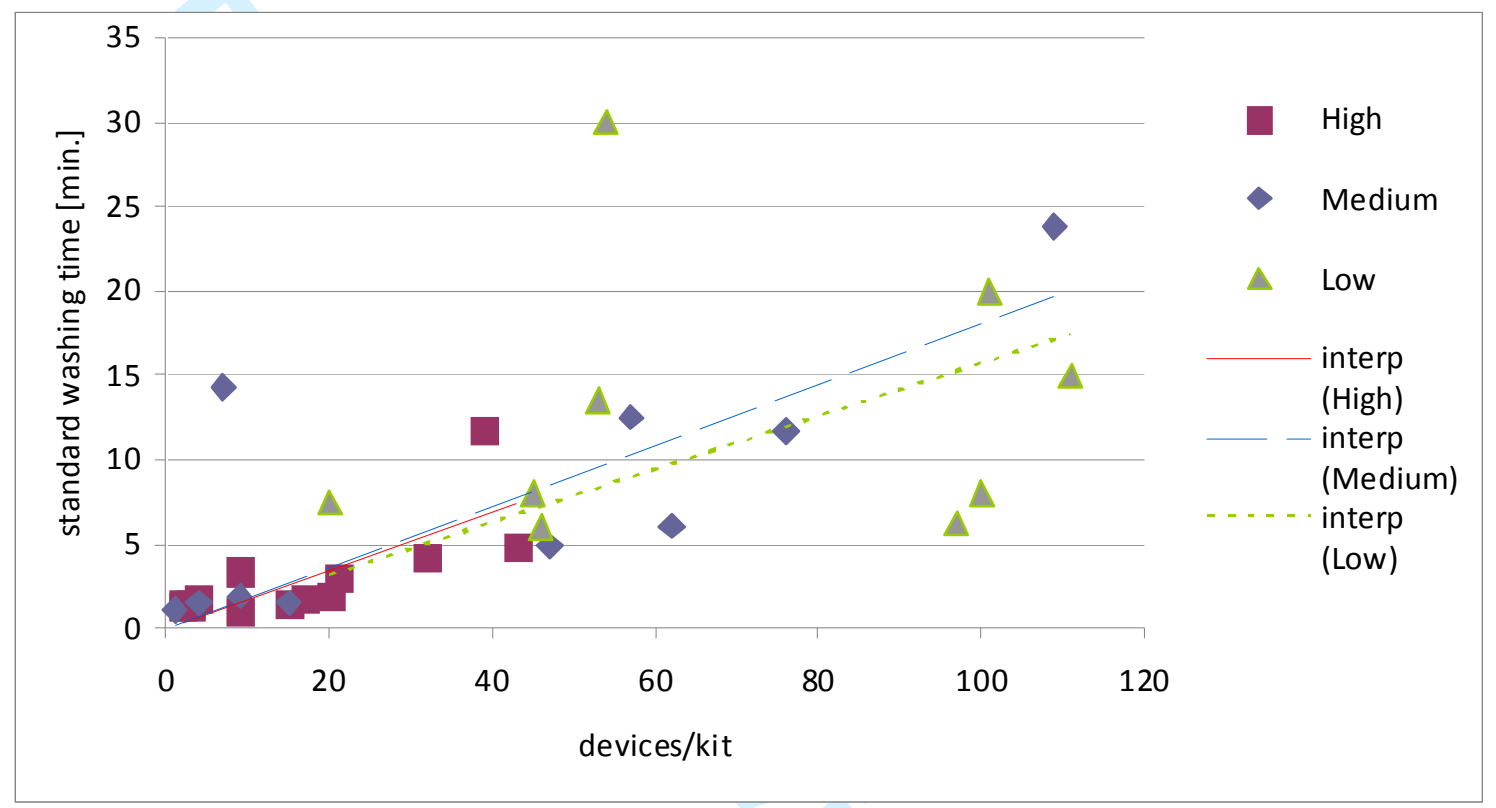

Figure 8 Standard time for the washing operation as a function of the number of devices included in each kit classified in three classes for handling difficulty.

The same analysis has been carried out in Figure 8 and Figure 9 and the resulting linear coefficients have been summarized in Table 4. 


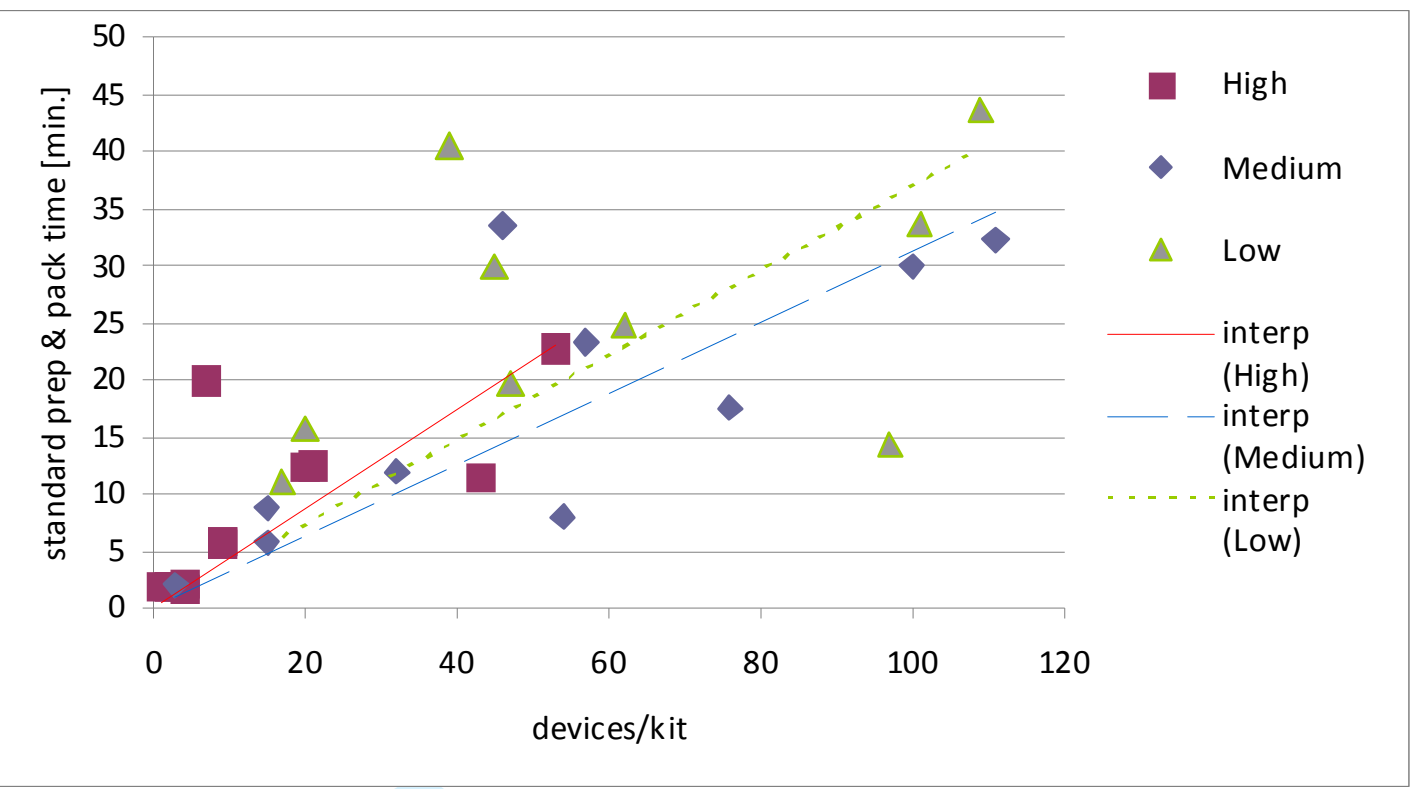

Figure 9 Standard time for the prep \& pack operation as a function of the number of devices included in each kit classified in three classes for handling difficulty.

Table 4 Standard time increase [minutes] per device in a kit for different dirt and handling difficulty levels on the two manual stages.

\begin{tabular}{cccc}
\hline Linear coefficient & $\begin{array}{c}\text { Washing dirt level } \\
\text { Figure 7 }\end{array}$ & $\begin{array}{c}\text { Washing difficulty } \\
\text { Figure 8 }\end{array}$ & $\begin{array}{c}\text { Prep \& pack difficulty } \\
\text { Figure 9 }\end{array}$ \\
\hline high & 0.211 & 0.171 & 0.436 \\
medium & 0.137 & 0.181 & 0.369 \\
low & 0.097 & 0.157 & 0.312 \\
\hline
\end{tabular}

From Table 4, the standard time increase per device in a new (unknown) kit for different dirt and handling difficulty levels on the two manual stages can be estimated. 
From equation (19), the plant setup is proportional to the actual setup time

$\left[\left(\sum_{i=1}^{N}\left(E_{k} \cdot s_{i j}\right) \cdot Z_{i j h b}\right) / v_{j}\right]$ obtained from the planned number $v_{j}$ of operators at the stage considered and increases with $E_{k}$ for performance degradation. Similarly, as shown in the application section for the line balancing in equation (23) and the plant capacity $\lambda_{\mathrm{w}}$ in equation (27).

The (highest) number of operators required for both stages determined with expression (24) is listed in Table 5 and their assignment has been evaluated by the scheduling algorithm in Rossi, Puppato and Lanzetta (2013).

Table 5 Results of the plant capacity analysis. Maxima are enhanced bold and in light grey for operators and dark grey for machines. Time windows on stage 2.

\begin{tabular}{|c|c|c|c|c|c|c|c|c|c|c|c|}
\hline \multirow[b]{2}{*}{ Delivery Time window } & \multicolumn{4}{|c|}{ Morning } & \multicolumn{6}{|c|}{ Afternoon } & \multirow{2}{*}{$\begin{array}{c}\text { Night } \\
\Delta_{11}\end{array}$} \\
\hline & $\Delta_{1}$ & $\Delta_{2}$ & $\Delta_{3}$ & $\Delta_{4}$ & $\Delta_{5}$ & $\Delta_{6}$ & $\Delta_{7}$ & $\Delta_{8}$ & $\Delta_{9}$ & $\Delta_{10}$ & \\
\hline Operators on stage $1\left(v_{1}\right)$ & 2 & 3 & 1 & 4 & 3 & 2 & 2 & 2 & 2 & 2 & 0 \\
\hline \multirow[t]{2}{*}{ Washing machine cycles } & 5 & 3 & 1 & 4 & 1 & 2 & 1 & 3 & 5 & 1 & 0 \\
\hline & $\Lambda_{1}$ & \multicolumn{3}{|c|}{$\Lambda_{2}$} & \multicolumn{3}{|c|}{$\Lambda_{3}$} & \multicolumn{3}{|c|}{$\Lambda_{4}$} & $\Lambda_{5}$ \\
\hline Operators on stage $2\left(V-v_{1}\right)$ & 0 & \multicolumn{3}{|c|}{5} & \multicolumn{3}{|c|}{4} & \multicolumn{3}{|c|}{4} & 1 \\
\hline Sterilization cycles & 0 & \multicolumn{3}{|c|}{5} & \multicolumn{3}{|c|}{3} & \multicolumn{3}{|c|}{3} & 4 \\
\hline
\end{tabular}

The results shown in Table 5 are based on peak day data with the kits arrivals in Figure 6 and assuming a machine capacity on the two stages of

- 3 large or 6 small trays for each of the 5 levels for washers and

- 24 medium containers for sterilizers,

assuming an average container size of 4 small trays. 
The average kit size $u_{j}$ is respectively 2 large (or 4 small) trays and 2 large (or 4 medium) containers.

The sum on the two stages of (4+5) plus 2 more operators dedicated to kits check-in and delivery is coincident with the average number in the actual sterilization plant (11).

Similarly, the number of machines on the two stages ( 5 and 5) is almost coincident to those actually available (6 and 5).

\section{Discussion}

According to the proposed hybrid direct observation/synthetic method, for the estimation of the standard activity time of new kits, the dirt level depends on the typical use of medical devices and can be more objectively determined from the belonging operating ward. The dirt level increases with time for the drying effect, which can be expressed as a function of the elapsed time from the release date.

As for the washing difficulty, the three classes investigated show small changes in the coefficient and even a wrong assignment at the medium and high levels. Consequently, in washing the dominating parameters remains the dirt level and only two classes for washing difficulty have been redefined with coefficients 0.157 and 0.176 . As for prep \& pack times, in addition of being generally higher, they are also strongly affected by the device number with a difficulty coefficient that more than doubles the washing coefficients.

It can be noticed that the simple capacity model presented predicts an amount of resources (operators and machines) confirmed by historical data, which were determined by qualitative estimations without detailed knowledge of the process and of manual activity times.

The operators requirements have been determined using the efficiency parameter $E_{k}$ in (3), which takes into account the performance degradation detailed in (22). Consequently, higher 
efficiency and lower operator requirements are expected by quality improvement, e.g. affecting parameters $\eta_{k}$ and $\mathrm{K}_{d}$.

It can also be stated that machines are sufficient for current needs and no investments are necessary.

For the sake of generalization of current work, the sterilization process may differ in other hospitals in term of routing, layout, number of stages etc. as described by the respective authors in the literature above, without affecting the transferability of the proposed operator efficiency model. Similarly the proposed efficiency model is transferable to other manufacturing and non manufacturing environments, where material products are concerned and process times are uncertain.

From a scientific viewpoint, the constraint of minimally affecting the system status quo has produced current operator efficiency model. In a manufacturing environment, standard times are determined by MTM or similar methods and become binding for operators; if activity times in a manufacturing system are not respected, productivity is affected up to the system collapse. The constraint of not changing the manufacturing system is typical of some labor situations as public organizations, where limited levers exist to enforce operators to adhere to the system specifications (e.g. achieving a minimum productivity level), so the opposite path has been followed. For the system design and run an operator model is necessary: in this approach a general model has been proposed to be tailored according to the actual operator's behavior.

A drawback of the proposed hybrid direct observation/synthetic method to standardize uncertain times is that a deeper understanding of governing factors is required to build the model and to increase its prediction accuracy, as opposed to direct observation methods, where only a greater amount of experimental data is required. A statistical analysis of the examined application has also been performed a posteriori for validation and could be applied for a sensitivity analysis on efficiency parameters. 
The operator efficiency parameters involve not only ergonomics, but also skill, stress, motivation, product being handled etc., with their non linear relationships. Despite the difficulty of assessing individual parameters, the model expression in (4) is quite simple and seems generally applicable also to other resource types, than operators.

The manufacturing-like case presented has been approached by an interdisciplinary analysis involving soft links to organization, quality, management, human factors, ergonomics etc. The usefulness of the analysis and of the proposed efficiency model is to show the connections of these disciplines with production planning. The system modules assessed are some examples of usual manufacturing modules to highlight the mentioned connections by the proposed efficiency parameter $E_{k}$. For human modeling extensive literature (not considered in this paper) is available to improve the model on ergonomics, motivation, fatigue etc.

In addition, the proposed operator model allows to predict the activity time as a result of various stochastic components and represents a framework to link production planning issues (layout design, resource assignment, scheduling, simulation, performance assessment), to human factors. These links can be further developed both with interdisciplinary contributions and by application to other contexts, than health services.

As for the method accuracy and cost, the activity time error in a direct observation method is a function of the number of observations, usually $\propto \frac{s}{\sqrt{O}}$ by (23) and their cost is $\propto O$.

Similarly the activity time error in a synthetic method decreases as a function of the number of coefficients in (4), while the model cost (combination of coefficients) usually increases exponentially with their number.

The application of proposed hybrid method requires a compromise between the number of direct observations on the field (e.g. on different operators and for different kits) and the operator model complexity described by its coefficients (e.g. skill, motivation, dirt level) in order to achieve the target accuracy at the minimum cost. 
As for the method transferability, the combination of synthetic parameters may reduce the total costs to estimate the activity times by direct observation. A major limitation is the knowledge and understanding of the main operator efficiency coefficients required in a synthetic method, while direct observation is straightforward.

\section{Conclusion}

Different approaches in the literature on uncertain activity times have been reviewed. In this work the effect of efficiency parameters on standardized activity times, defined according to industry practice, has been considered. A simple and general operator efficiency model has been proposed and an application has been described in detail, to show how various typical manufacturing activities, such as production planning, plant setup, capacity analysis, operator assignment and scheduling, are affected by uncertain activity times.

The proposed model can be applied as is, with special care on the degree of detail in the efficiency parameter, in order to achieve a compromise between the operator modeling effort (which may be significant) and the economical benefits from the improved production planning (resource utilization, tardy jobs, makespan etc.).

The proposed approach represents an example application of Method Time Measurement transferred to healthcare with extension potential to a number of also non manufacturing cases, such as: transportation, food processing/restaurants, agriculture, document processing/administration, services, sport competitions and many others.

Regarding the direct observation component of the activity time, the operator performance change as modeled in this paper can be the input of future theoretical and applied research, respectively in combination of stochastic approaches and in heuristics and metaheuristics optimization with uncertain times. As for the synthetic component, recent advances of digital 
human models and virtual simulation represent an alternative source of data; this application of the proposed hybrid direct observation/synthetic approach requires further investigation. Considering other resource types than operators, an extension of current hybrid method is modeling of machines efficiency, like robots in extreme conditions (in space, underwater) or manufacturing plants in real conditions, involving the analysis of interdisciplinary parameters such as environment, diagnosis, maintenance, aging etc.

\section{Acknowledgements}

The authors would like to thank Dr. Carlo R. Tomassini, Executive Director, and Dr. Marco Nerattini, Head of Operating Unit Innovation, development and process analysis and the technical staff of the Central Sterile Services Department (CSSD).

\section{References}

Ahmadizar, F., M. Ghazanfari, and S. M. T. Fatemi Ghomi. 2010. "Group shops scheduling with makespan criterion subject to random release dates and processing times." Computers \& Operations Research 37 (1): 152-162.

Arnaout, J. P. 2014. "Rescheduling of parallel machines with stochastic processing and setup times.” Journal of Manufacturing Systems 33 (3): 376-384 doi:10.1016/j.jmsy.2014.02.003.

Aytug, H., M. A. Lawley, K. McKay, S. Mohan, and R. Uzsoy. 2005. "Executing production schedules in the face of uncertainties: A review and some future directions." European Journal of Operational Research 161 (1): 86-110.

Balasubramanian, J., and I. E. Grossmann. 2002. “A novel branch and bound algorithm for scheduling flowshop plants with uncertain processing times." Computers \& chemical engineering 26 (1): 41-57. 
Becker, C., and A. Scholl. 2006. "A survey on problems and methods in generalized assembly line balancing." European Journal of Operational Research 168 (3), 1 February 2006: 694715. doi:10.1016/j.ejor.2004.07.023.

Bedny, G. Z., W. Karwowski and F. Voskoboynikov. 2015. “Application of Standardized Motions in Temporal Analysis of Work Activity." Human Factors and Ergonomics in Manufacturing \& Service Industries 25: 469-483. doi: 10.1002/hfm.20562

Buxey, G. M., N. D. Slack, and R. Wild. 1973. "Production Flow Line System Design-A Review." A I I E Transactions 5 (1): 37-48.

Chenhall, R. H. 2003. "Management control systems design within its organizational context: findings from contingency-based research and directions for the future." Accounting, organizations and society 28 (2): 127-168.

Choi, S. H., and K. Wang. 2012. "Flexible flow shop scheduling with stochastic processing times: A decomposition-based approach.” Computers \& Industrial Engineering 63 (2): 362373.

Dale, B. G., T. Van Der Wiele, and J. Van Iwaarden. 2013. Managing quality. John Wiley \& Sons.

Dallery, Y., and S. B. Gershwin. 1992. "Manufacturing flow line systems: a review of models and analytical results." Queueing Systems 12 (1): 3-94. doi:10.1007/BF01158636.

Di Mascolo, M., and A. Gouin. 2013. “A generic simulation model to assess the performance of sterilization services in health establishments." Health care management science 16 (1): 45-61.

Eshtehardian, E., A. Afshar, and R. Abbasnia. 2009. "Fuzzy-based MOGA approach to stochastic time-cost trade-off problem." Automation in Construction 18 (5): 692-701.

Garey, M. R., and D. S. Johnson. 1979. Computers and intractability: A guide to the theory of NP-completeness. San Francisco: W.H. Freeman and Company.

Gourgand, M., N. Grangeon, and S. Norre. 2003. "A contribution to the stochastic flow shop scheduling problem.” European Journal of Operational Research, 151 (2): 415-433.

Hao, X., L. Lin, and M. Gen. 2014. "An Effective Multi-objective EDA for Robust Resource Constrained Project Scheduling with Uncertain Durations.” Procedia Computer Science 36: $571-578$. 
Hapke, M., A. Jaszkiewicz, and R. Slowinski. 1994. "Fuzzy project scheduling system for software development." Fuzzy sets and systems 67 (1): 101-117.

Henrique, D. B., A. F. Rentes, M. Godinho Filho, and K. F. Esposto. 2016. "A new value stream mapping approach for healthcare environments." Production Planning \& Control 27 (1): 24-48.

ISO 9004. 2009. "Managing for the sustained success of an organization -- A quality management approach.”

ISO 17665-1. 2006. "Sterilization of health care products - Moist heat - Part 1: Requirements for the development, validation and routine control of a sterilization process for medical devices."

Kalhor, E., M. Khanzadi, E. Eshtehardian, and A. Afshar. 2011. "Stochastic time-cost optimization using non-dominated archiving ant colony approach." Automation in Construction 20 (8): 1193-1203.

Ke, H., and B. Liu. 2005. "Project scheduling problem with stochastic activity duration times." Applied Mathematics and Computation 168 (1): 342-353.

Krueger, D. C., M. Mellat Parast, and S. Adams. 2014. "Six Sigma implementation: a qualitative case study using grounded theory.” Production Planning \& Control 25 (10): 873889.

Kuo, C. F., and M. J. Wang. 2009. "Motion generation from MTM semantics.” Computers in Industry 60 (5): 339-348.

Larsson, A. 2013. "The accuracy of surgery time estimations." Production Planning \& Control 24 (10-11): 891-902.

Ozturk, O., M. A. Begen, and G. S. Zaric. 2014. "A branch and bound based heuristic for makespan minimization of washing operations in hospital sterilization services." European Journal of Operational Research 239 (1): 214-226. doi:10.1016/j.ejor.2014.05.014.

Ozturk, O., M. L. Espinouse, M. Di Mascolo, and A. Gouin. 2010. "Optimizing the makespan of washing operations of medical devices in hospital sterilization services." IEEE Workshop on Health Care Management (WHCM): 1-6.

Ozturk, O., M. L. Espinouse, M. Di Mascolo, and A. Gouin. 2012. "Makespan minimisation on parallel batch processing machines with non-identical job sizes and release dates." International Journal of Production Research 50 (20): 6022-6035. 
Petrovic, S., and X. Song. 2006. “A new approach to two-machine flow shop problem with uncertain processing times." Optimization and Engineering 7(3): 329-342.

Piercy, N., W. Phillip, and M. Lewis. 2012. "Change management in the public sector: the use of cross-functional teams." Production Planning \& Control 24 (10-11): 976-987. doi:10.1080/09537287.2012.666913

Razmi, J., and M. Shakhs-Niyaee. 2008. "Developing a specific predetermined time study approach: an empirical study in a car industry." Production Planning \& Control 19 (5): 454460.

Reymondon, F., B. Pellet, and E. Marcon. 2008. "Optimization of hospital sterilization costs proposing new grouping choices of medical devices into packages." International Journal of Production Economics 112 (1): 326-335.

Rossi, A. 2014. "Flexible job shop scheduling with sequence-dependent setup and transportation times by ant colony with reinforced pheromone relationships." International Journal of Production Economics 153: 253-267. doi:10.1016/j.ijpe.2014.03.006.

Rossi, A., and M. Lanzetta. 2014. "Native metaheuristics for non-permutation flowshop scheduling." Journal of Intelligent Manufacturing 27 (4): 349-357. doi:10.1007/s10845-0120724-8.

Rossi, A., A. Pandolfi, and M. Lanzetta. 2014. "Dynamic priority for hybrid flowshop scheduling with parallel batching machines.” International Journal of Production Research 52 (13): 3842-3857. doi:10.1080/00207543.2013.835496.

Rossi, A., A. Puppato, and M. Lanzetta. 2013. "Heuristics for scheduling a two-stage hybrid flow shop with parallel batching machines: Application at a hospital sterilisation plant." International Journal of Production Research 51 (8): 2363-2376. doi:10.1080/00207543.2012.737942.

Rossi, A., S. Soldani, and M. Lanzetta. 2015. "Hybrid stage shop scheduling." Expert Systems with Applications 42 (8): 4105-4119. doi:10.1016/j.eswa.2014.12.050.

Stecke, K. E., and N. Raman. 1995. "FMS Planning Decision, Operating Flexibilities and System Performance.” IEEE Transaction on Engineering Management 42 (1): 82-90.

Swaminathan, R., M. E. Pfund, J. W. Fowler, S. J. Mason, and A. Keha. 2007. "Impact of permutation enforcement when minimizing total weighted tardiness in dynamic flowshops with uncertain processing times." Computers \& Operations Research 34 (10): 3055-3068. 
van de Klundert, J., P. Muls, and M. Schadd. 2008. "Optimizing sterilization logistics in hospitals." Health care management science 11 (1): 23-33.

\section{Appendix}

Table 6 Time windows for kit arrival at the sterilization plant.

\begin{tabular}{|l|r|l|r|r|r|}
\hline From & $7: 30$ & To & $10: 30$ & $\Delta_{1}$ & $180 \mathrm{~min}$. \\
\hline From & $10: 30$ & To & $11: 30$ & $\Delta_{2}$ & $90 \mathrm{~min}$. \\
\hline From & $11: 30$ & To & $12: 30$ & $\Delta_{3}$ & $60 \mathrm{~min}$. \\
\hline From & $12: 30$ & To & $14: 00$ & $\Delta_{4}$ & $90 \mathrm{~min}$. \\
\hline From & $14: 00$ & To & $14: 30$ & $\Delta_{5}$ & $90 \mathrm{~min}$. \\
\hline From & $14: 30$ & To & $15: 30$ & $\Delta_{6}$ & $60 \mathrm{~min}$. \\
\hline From & $15: 30$ & To & $16: 30$ & $\Delta_{7}$ & $60 \mathrm{~min}$. \\
\hline From & $16: 30$ & To & $18: 00$ & $\Delta_{8}$ & $90 \mathrm{~min}$. \\
\hline From & $18: 00$ & To & $19: 30$ & $\Delta_{9}$ & $90 \mathrm{~min}$. \\
\hline From & $19: 30$ & To & $21: 00$ & $\Delta_{10}$ & $90 \mathrm{~min}$. \\
\hline From & $21: 00$ & To & $7: 30$ & $\Delta_{11}$ & $630 \mathrm{~min}$. \\
\hline
\end{tabular}

Table 7 Time windows extrapolated on the second stage.

\begin{tabular}{|l|r|l|r|r|r|}
\hline From & $7: 30$ & To & $10: 40$ & $\Lambda_{\mathrm{M} 1}$ & $190 \mathrm{~min}$. \\
\hline From & $10: 40$ & To & $14: 00$ & $\Lambda_{\mathrm{M} 2}$ & $200 \mathrm{~min}$. \\
\hline From & $14: 00$ & To & $17: 30$ & $\Lambda_{\mathrm{P} 1}$ & $210 \mathrm{~min}$. \\
\hline From & $17: 30$ & To & $21: 00$ & $\Lambda_{\mathrm{P} 2}$ & $210 \mathrm{~min}$. \\
\hline From & $21: 00$ & To & $7: 30$ & $\Lambda_{\mathrm{N}}$ & $810 \mathrm{~min}$. \\
\hline
\end{tabular}


Table 8 Measured washing and prep \& pack times expressed in minutes, with their mean $s_{i j}$ and standard deviation $s$.

\begin{tabular}{|c|c|c|c|c|c|c|c|c|}
\hline Kit & \multicolumn{2}{|c|}{ Cataract } & \multicolumn{2}{|c|}{ Thyroid } & \multicolumn{2}{|c|}{ Intestinal surgery } & \multicolumn{2}{|c|}{ Faco ozil } \\
\hline & $\begin{array}{c}\text { Washing } \\
\text { times }\end{array}$ & $\begin{array}{c}\text { Prep \& } \\
\text { pack times }\end{array}$ & $\begin{array}{c}\text { Washing } \\
\text { times }\end{array}$ & $\begin{array}{c}\text { Prep \& } \\
\text { pack times }\end{array}$ & $\begin{array}{c}\text { Washing } \\
\text { times }\end{array}$ & $\begin{array}{c}\text { Prep \& } \\
\text { pack times }\end{array}$ & $\begin{array}{c}\text { Washing } \\
\text { times }\end{array}$ & $\begin{array}{c}\text { Prep \& } \\
\text { pack times }\end{array}$ \\
\hline 1 & 0.40 & 3.57 & 9.15 & 7.47 & 7.40 & 6.50 & 0.37 & 1.01 \\
\hline 2 & 0.41 & 3.42 & 5.29 & 5.28 & 12.36 & 4.41 & 1.11 & 0.42 \\
\hline 3 & 0.41 & 3.23 & 7.38 & 5.20 & 15.43 & 4.25 & 0.58 & 0.58 \\
\hline 4 & 0.55 & 3.04 & 5.31 & 5.35 & 11.17 & 3.21 & 0.43 & 0.59 \\
\hline 5 & 1.08 & 3.18 & 4.12 & 5.04 & 7.51 & 4.06 & 0.50 & 0.39 \\
\hline 6 & 1.09 & 3.28 & 5.17 & 4.00 & 11.09 & 4.18 & 0.52 & 1.05 \\
\hline 7 & 0.56 & 2.21 & 6.29 & 3.22 & 10.48 & 4.22 & 0.56 & 0.34 \\
\hline 8 & 0.45 & 2.32 & 5.03 & 4.51 & 7.39 & 5.09 & 1.27 & 1.05 \\
\hline 9 & 1.08 & 3.24 & 5.53 & 4.30 & 8.06 & 3.15 & 0.35 & 1.19 \\
\hline 10 & 1.16 & 2.38 & 4.02 & 5.24 & 16.26 & 3.29 & 0.41 & 0.35 \\
\hline 11 & 1.03 & 4.19 & 5.37 & 7.15 & 14.16 & 3.53 & 1.05 & 0.37 \\
\hline 12 & 1.13 & 2.46 & 4.27 & 5.26 & 17.59 & 4.57 & 0.30 & 0.53 \\
\hline$s_{i j}$ & 0.78 & 3.04 & 5.58 & 5.17 & 11.58 & 4.21 & 0.62 & 0.66 \\
\hline$s$ & 0.33 & 0.59 & 1.46 & 1.19 & 3.64 & 0.94 & 0.33 & 0.32 \\
\hline
\end{tabular}

URL: http://mc.manuscriptcentral.com7tppc E-mail: ppc@plymouth.ac.uk 


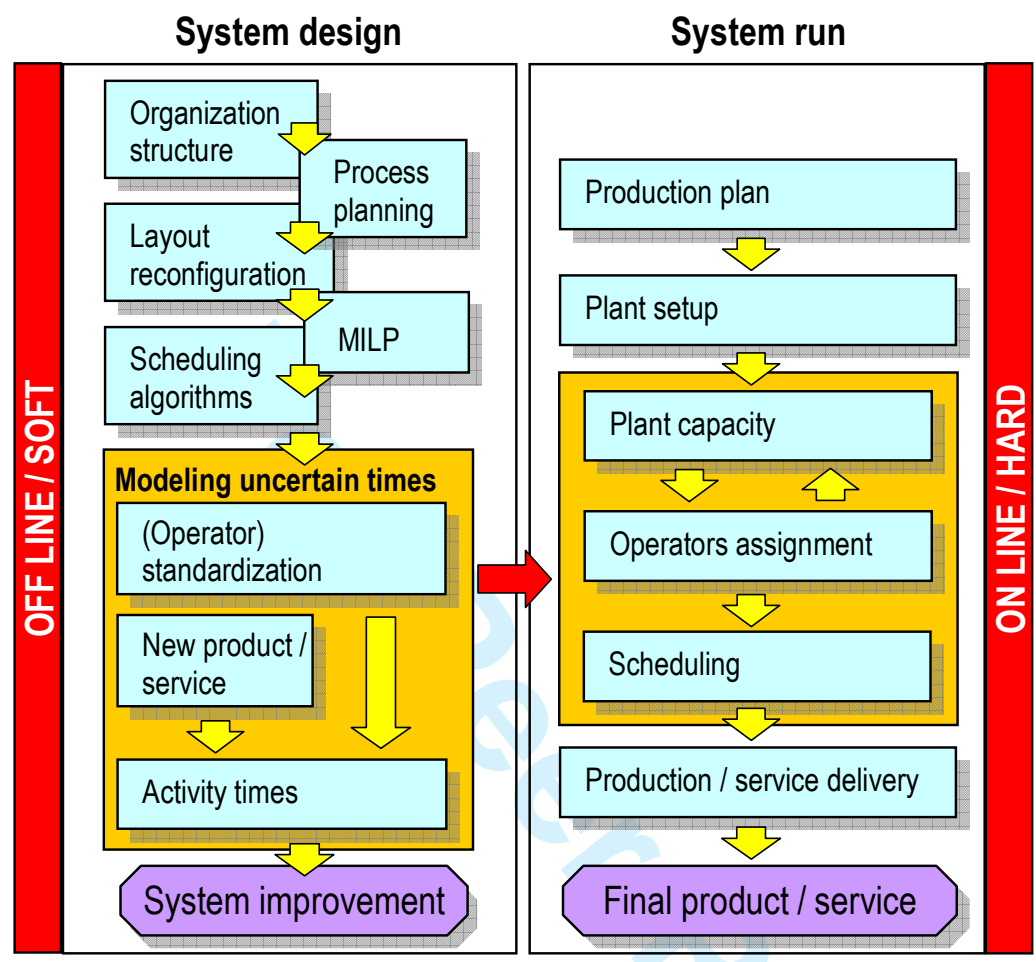




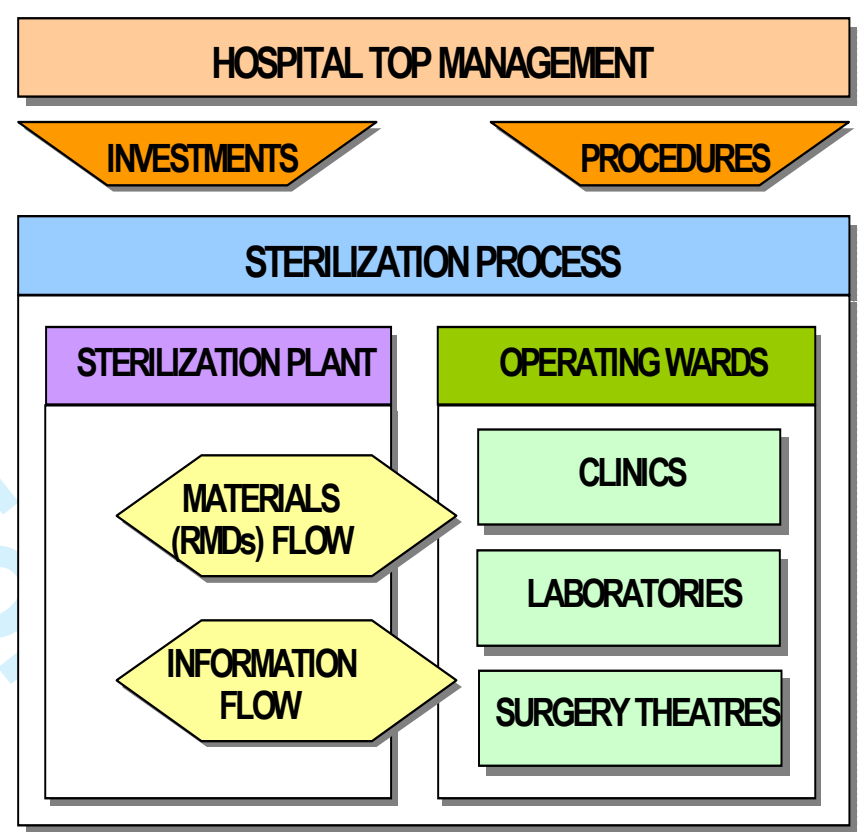

Figure 3 The main material and immaterial flows involved in the sterilization process, which integrates and impacts most hospital operating wards.

Improvement is driven by the top management. 


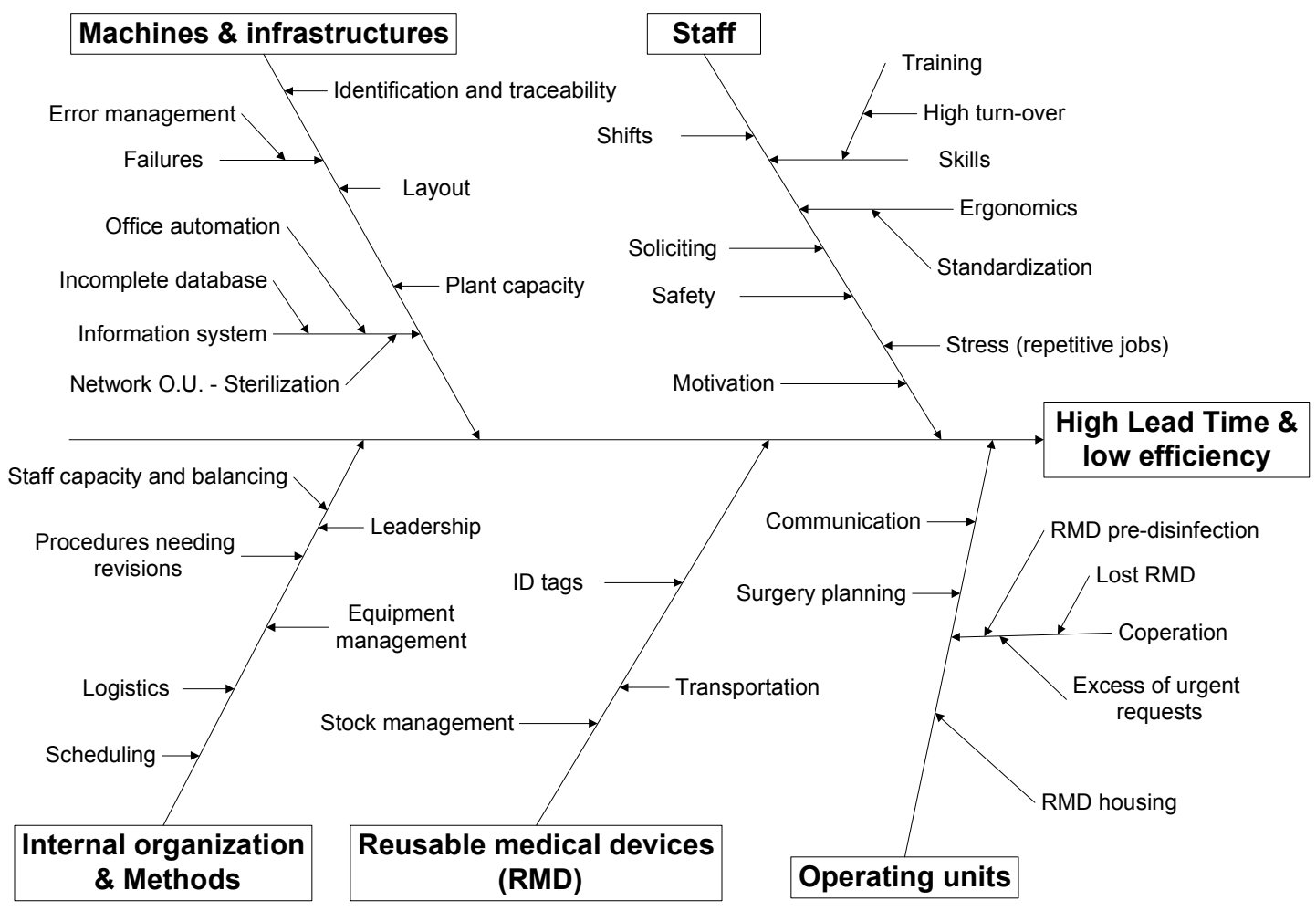

Figure 4 Overview of the main improvement areas of the sterilization plant at the examined AOUP hospital. 


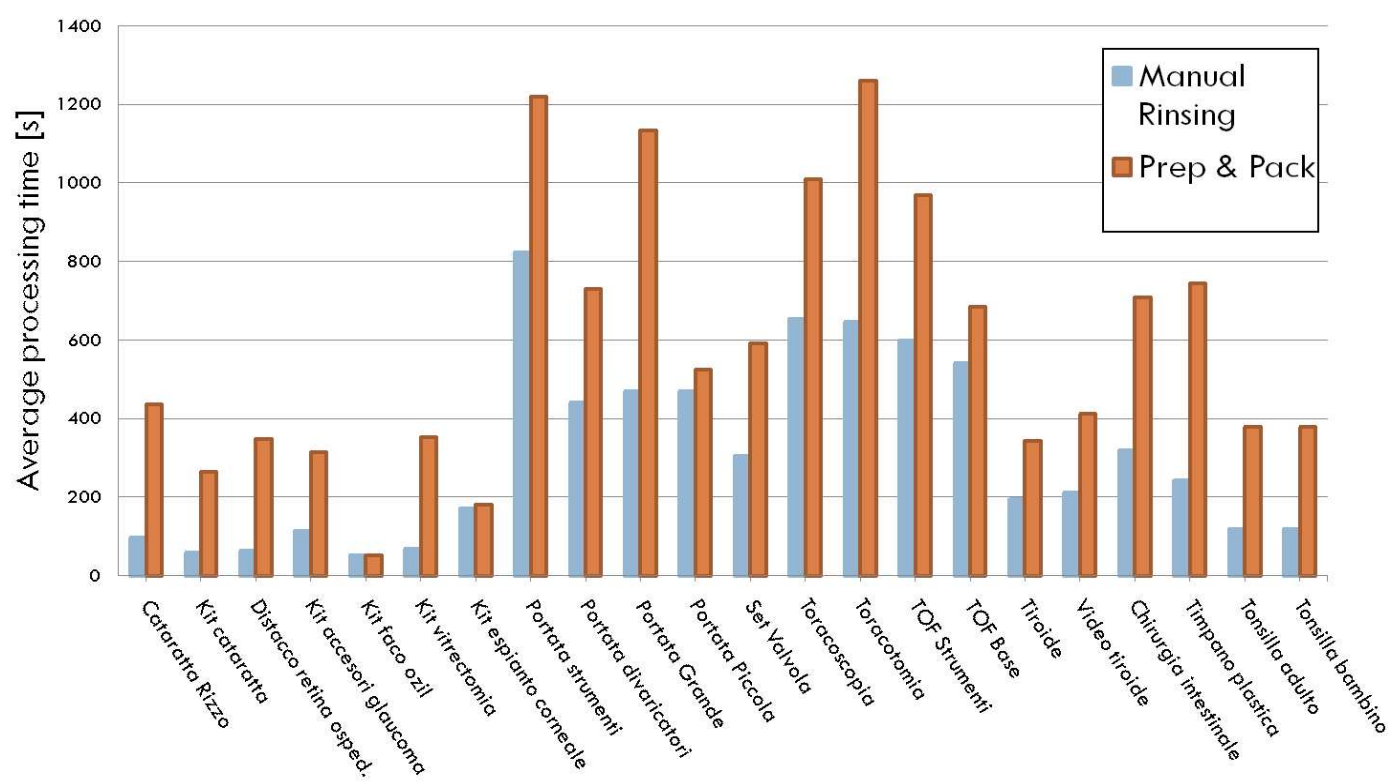

Figure 5 Standard processing times $s_{i j}$ in the two manual operations compared. 


\section{Surgery} theaters

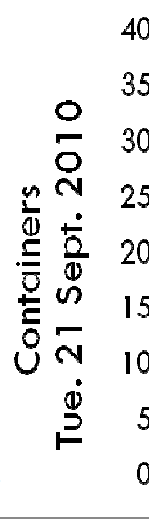

40

\begin{tabular}{|c|c|c|c|c|c|c|c|c|c|c|c|}
\hline \multicolumn{12}{|l|}{40} \\
\hline \multirow{7}{*}{ Surgery } & & & & & & & & & 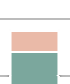 & & \\
\hline & & & & & & & & & 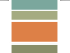 & & \\
\hline & & & & & & & & & & & \\
\hline & & & & & & & & $=$ & & & \\
\hline & & & & & & & & & & & \\
\hline & & & & & & & & & & & \\
\hline & & & & & 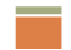 & & $=$ & & & E & \\
\hline theaters & 7.30 & 10.30 & 11.30 & 12.30 & 14.00 & 14.30 & 15.30 & 16.30 & 18.00 & 19.30 & 21.00 \\
\hline CHIRURGIAGBNERALE $4^{\circ}$ - 97 & 0 & 0 & 0 & 4 & 0 & 1 & 0 & 1 & 0 & 0 & 0 \\
\hline -CHIRURG|ASENOLOGICA - 48 & 0 & 0 & 0 & 0 & 0 & 0 & 0 & 0 & 0 & 0 & 0 \\
\hline CHIRURGIA VASCOLARE - 43 & 1 & 0 & 0 & 0 & 0 & 0 & 1 & 0 & 2 & 0 & 0 \\
\hline - CHIRURGIA GENERALE - 42 & 6 & 0 & 0 & 0 & 0 & 0 & 0 & 0 & 3 & 1 & 0 \\
\hline - CHIRURGIA TORACICA - 02 & 2 & 0 & 0 & 0 & 1 & 0 & 1 & 0 & 1 & 0 & 0 \\
\hline CARDIOCHIRURGIA-01 & 1 & 0 & 0 & 0 & 3 & 0 & 0 & 0 & 2 & 0 & 0 \\
\hline -UROLOGIAUNIVERSITARIA - 125 & 2 & 0 & 0 & 0 & 0 & 4 & 0 & 1 & 0 & 2 & 0 \\
\hline -UROLOGIA OSPEDALLERA - 109 & 12 & 2 & 1 & 1 & 0 & 0 & 0 & 1 & 0 & 0 & 0 \\
\hline - CHIRURGIAGEN. $2^{\circ}$ - 124 & 5 & 3 & 0 & 4 & 0 & 4 & 0 & 5 & 0 & 1 & 0 \\
\hline - CHRURGIAGEN $4^{\circ}$ - 124 & 0 & 0 & 0 & 0 & 0 & 0 & 0 & 0 & 0 & 0 & 0 \\
\hline -OTORINO - 101 & 4 & 2 & 0 & 3 & 0 & 2 & 0 & 1 & 6 & 0 & 0 \\
\hline - CHIRURGIA GEN. COLON RETALE - 10 & 07 & 3 & 0 & 3 & 0 & 6 & 0 & 0 & 0 & 0 & 0 \\
\hline DOCUISTICA - 106 & 0 & 10 & 0 & 10 & 0 & 1 & $a$ & 8 & 20 & 0 & 0 \\
\hline
\end{tabular}

Figure 6 Kits per surgery theatre and time window on a peak date. 


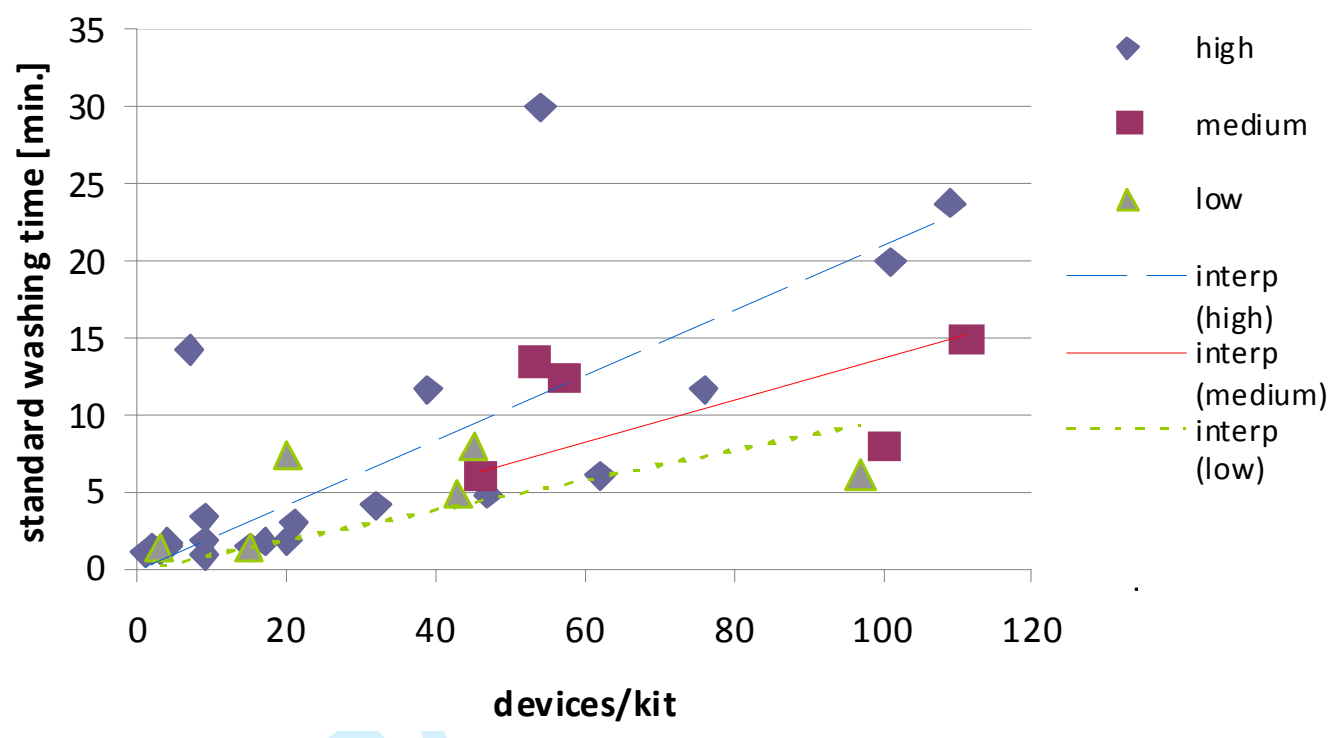

Figure 7 Standard time for the washing operation as a function of the number of devices included in each kit classified in three classes for presence of dirt. 


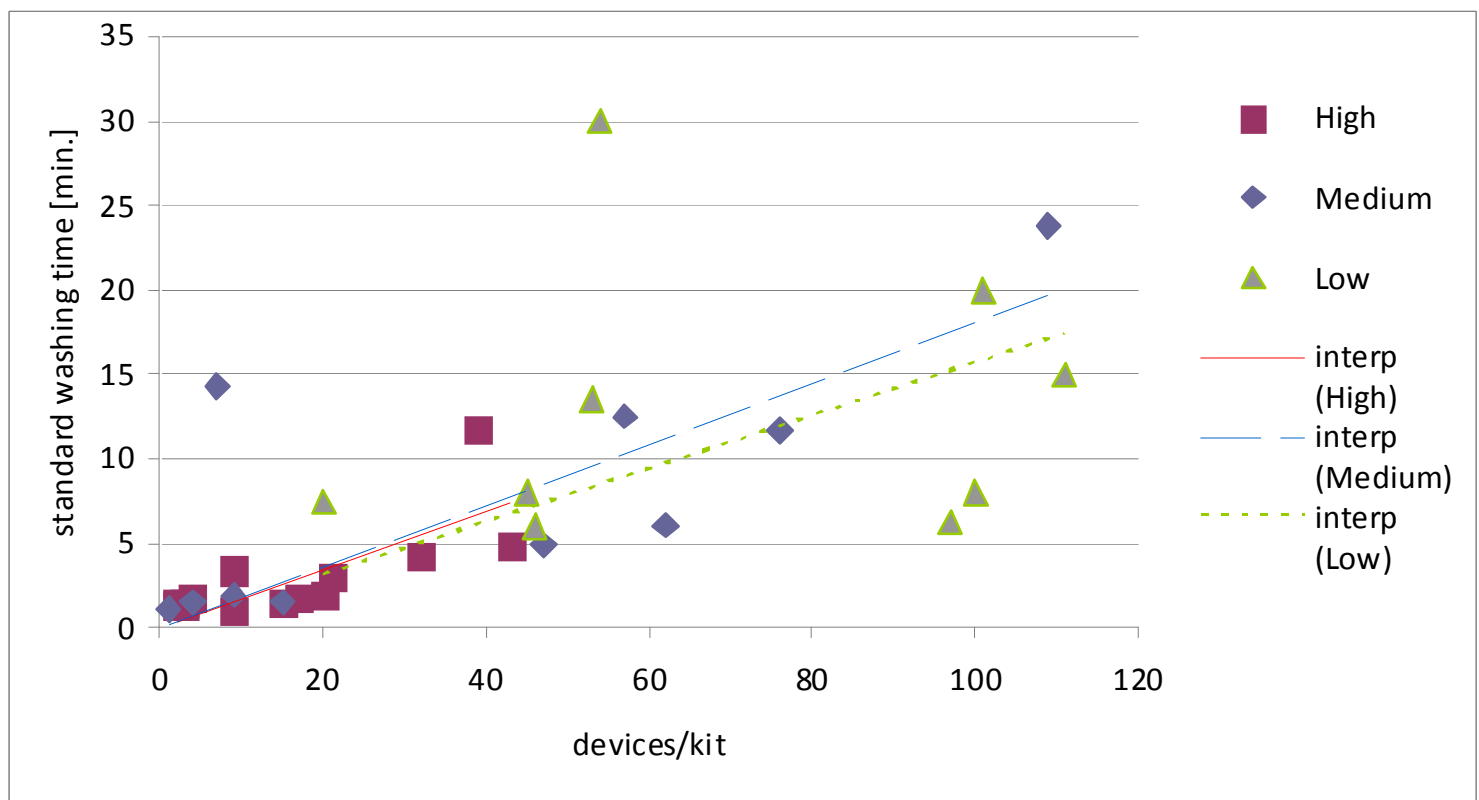

Figure 8 Standard time for the washing operation as a function of the number of devices included in each kit classified in three classes for handling difficulty. 


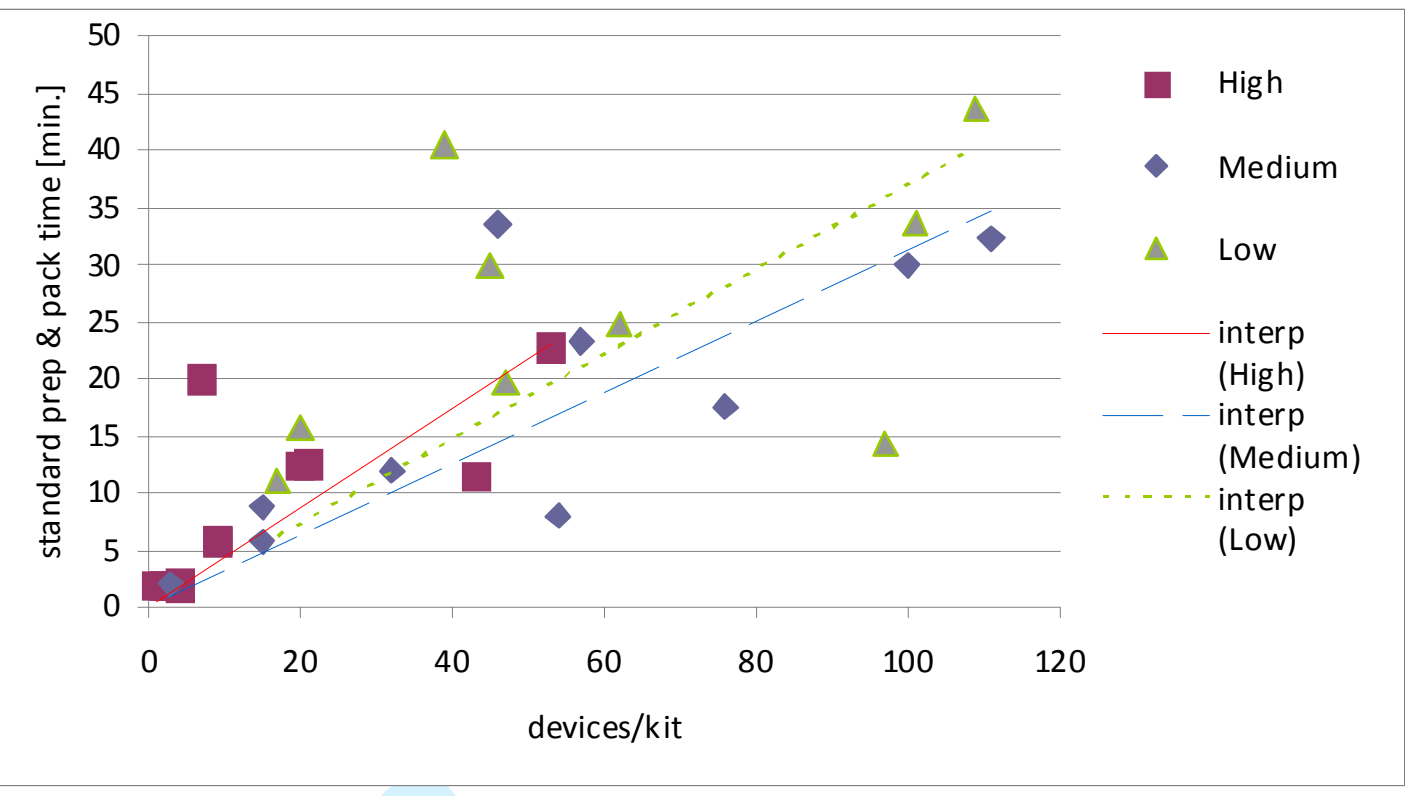

Figure 9 Standard time for the prep \& pack operation as a function of the number of devices included in each kit classified in three classes for handling difficulty. 


\begin{tabular}{|c|c|c|c|}
\hline \multicolumn{4}{|c|}{$\begin{array}{l}\text { STERILIZATION PROCESS PLAN } \\
\text { AOUP hospital }\end{array}$} \\
\hline $\begin{array}{l}\text { Kit name: } \\
\text { Thyroid }\end{array}$ & & Ward: General surgery & $\begin{array}{l}\text { Sheet } \\
1 \text { of } 1\end{array}$ \\
\hline $\begin{array}{l}\text { Updated: } \\
21 / 09 / 10\end{array}$ & & Priority: $\square$ normal $-\square$ life saving & \\
\hline Stage $j$ & Phase & Subphase & $\begin{array}{l}\text { Time } \\
\text { [min.] } s_{i j}\end{array}$ \\
\hline & 10 & 10.1 - kits check-in & \\
\hline $\begin{array}{l}\text { Setup } \\
\text { (washing) }\end{array}$ & 20 & $\begin{array}{l}20.2 \text { - manual rinsing } \\
20.3 \text { - medical devices loading on trays } \\
20.4 \text { - tray loading on wash cart } \\
20.5 \text { - cart loading on washing machine to } \\
\text { specified machine capacity (batch } \\
\text { forming and close) }\end{array}$ & 4.9 \\
\hline 8 & 30 & 30.1 - mechanical washing & 70 \\
\hline $\begin{array}{l}\text { Setup } \\
\text { (prep \& } \\
\text { pack) }\end{array}$ & 40 & $\begin{array}{l}40.1 \text { - cart and tray unloading } \\
40.2 \text { - medical devices visual inspection } \\
40.3 \text { - medical devices preparation } \\
40.4 \text { - list check } \\
40.5 \text { - packing into containers } \\
40.6 \text { - containers loading into autoclave to } \\
\text { specified machine capacity (batch } \\
\text { forming and close) }\end{array}$ & 11.5 \\
\hline 2 & 50 & $50.1-$ steam sterilization & 70 \\
\hline & 60 & $\begin{array}{l}60.1 \text { - containers unloading } \\
60.2 \text { - kits forming } \\
60.3 \text { - shipping }\end{array}$ & \\
\hline
\end{tabular}




\begin{tabular}{|c|c|c|c|c|}
\hline \multirow{2}{*}{ Normality test / kit name } & Cataract & Thyroid & $\begin{array}{c}\text { Intestinal } \\
\text { surgery }\end{array}$ & Faco ozil \\
\cline { 2 - 5 } & \multicolumn{4}{|c|}{ P-value } \\
\hline Shapiro - Wilk & 0.12 & 0.91 & 0.29 & 0.75 \\
\hline Lilliefors & 0.27 & 0.88 & 0.03 & 0.65 \\
\hline Jarque - Bera & 0.55 & 0.82 & 0.72 & 0.86 \\
\hline Cramer - Von Mises & 0.20 & 0.82 & 0.10 & 0.82 \\
\hline Anderson - Darling & 0.15 & 0.87 & 0.14 & 0.87 \\
\hline
\end{tabular}




\begin{tabular}{|c|c|c|c|c|}
\hline \multirow{2}{*}{ Normality test / kit name } & Cataract & Thyroid & $\begin{array}{c}\text { Intestinal } \\
\text { surgery }\end{array}$ & Faco ozil \\
\cline { 2 - 5 } & \multicolumn{3}{|c|}{ P-value } \\
\hline Shapiro - Wilk & 0.13 & 0.11 & 0.21 & 0.28 \\
\hline Lilliefors & 0.55 & 0.15 & 0.38 & 0.39 \\
\hline Jarque - Bera & 0.17 & 0.24 & 0.63 & 0.27 \\
\hline Cramer - Von Mises & 0.39 & 0.13 & 0.40 & 0.26 \\
\hline Anderson - Darling & 0.25 & 0.13 & 0.30 & 0.27 \\
\hline
\end{tabular}


Linear coefficient Washing dirt level Washing difficulty Prep \& pack difficulty Figure 7

Figure 8

\begin{tabular}{cccc}
\hline high & 0.211 & 0.171 & 0.436 \\
medium & 0.137 & 0.181 & 0.369 \\
low & 0.097 & 0.157 & 0.312 \\
\hline
\end{tabular}




\begin{tabular}{|c|c|c|c|c|c|c|c|c|c|c|c|}
\hline \multirow[b]{2}{*}{ Delivery Time window } & \multicolumn{4}{|c|}{ Morning } & \multicolumn{6}{|c|}{ Afternoon } & \multirow{2}{*}{$\begin{array}{c}\text { Night } \\
\Delta_{11}\end{array}$} \\
\hline & $\Delta_{1}$ & $\Delta_{2}$ & $\Delta_{3}$ & $\Delta_{4}$ & $\Delta_{5}$ & $\Delta_{6}$ & $\Delta_{7}$ & $\Delta_{8}$ & $\Delta_{9}$ & $\Delta_{10}$ & \\
\hline Operators on stage $1\left(v_{1}\right)$ & 2 & 3 & 1 & 4 & 3 & 2 & 2 & 2 & 2 & 2 & 0 \\
\hline \multirow[t]{2}{*}{ Washing machine cycles } & 5 & 3 & 1 & 4 & 1 & 2 & 1 & 3 & 5 & 1 & 0 \\
\hline & $\Lambda_{1}$ & \multicolumn{3}{|c|}{$\Lambda_{2}$} & \multicolumn{3}{|c|}{$\Lambda_{3}$} & \multicolumn{3}{|c|}{$\Lambda_{4}$} & $\Lambda_{5}$ \\
\hline Operators on stage $2\left(V-v_{1}\right)$ & 0 & \multicolumn{3}{|c|}{5} & \multicolumn{3}{|c|}{4} & \multicolumn{3}{|c|}{4} & 1 \\
\hline Sterilization cycles & 0 & \multicolumn{3}{|c|}{5} & \multicolumn{3}{|c|}{3} & \multicolumn{3}{|c|}{3} & 4 \\
\hline
\end{tabular}




\begin{tabular}{|c|c|c|c|c|c|}
\hline From & $7: 30$ & To & $10: 30$ & $\Delta_{1}$ & $180 \mathrm{~min}$. \\
\hline From & $10: 30$ & To & $11: 30$ & $\Delta_{2}$ & $90 \mathrm{~min}$. \\
\hline From & $11: 30$ & To & $12: 30$ & $\Delta_{3}$ & $60 \mathrm{~min}$. \\
\hline From & $12: 30$ & To & $14: 00$ & $\Delta_{4}$ & $90 \mathrm{~min}$. \\
\hline From & $14: 00$ & To & $14: 30$ & $\Delta_{5}$ & $90 \mathrm{~min}$. \\
\hline From & $14: 30$ & To & $15: 30$ & $\Delta_{6}$ & $60 \mathrm{~min}$. \\
\hline From & $15: 30$ & To & $16: 30$ & $\Delta_{7}$ & $60 \mathrm{~min}$. \\
\hline From & $16: 30$ & To & $18: 00$ & $\Delta_{8}$ & $90 \mathrm{~min}$. \\
\hline From & 18:00 & To & 19:30 & $\Delta_{9}$ & $90 \mathrm{~min}$. \\
\hline From & 19:30 & To & 21:00 & $\Delta_{10}$ & $90 \mathrm{~min}$. \\
\hline From & 21:00 & To & 7:30 & $\Delta_{11}$ & $630 \mathrm{~min}$. \\
\hline
\end{tabular}

30 


\begin{tabular}{|l|r|r|r|r|r|}
\hline From & $7: 30$ & To & $10: 40$ & $\Lambda_{\mathrm{M} 1}$ & $190 \mathrm{~min}$. \\
\hline From & $10: 40$ & To & $14: 00$ & $\Lambda_{\mathrm{M} 2}$ & $200 \mathrm{~min}$. \\
\hline From & $14: 00$ & To & $17: 30$ & $\Lambda_{\mathrm{P} 1}$ & $210 \mathrm{~min}$. \\
\hline From & $17: 30$ & To & $21: 00$ & $\Lambda_{\mathrm{P} 2}$ & $210 \mathrm{~min}$. \\
\hline From & $21: 00$ & To & $7: 30$ & $\Lambda_{\mathrm{N}}$ & $810 \mathrm{~min}$. \\
\hline
\end{tabular}




\begin{tabular}{|c|c|c|c|c|c|c|c|c|}
\hline Kit & \multicolumn{2}{|c|}{ Cataract } & \multicolumn{2}{|c|}{ Thyroid } & \multicolumn{2}{|c|}{ Intestinal surgery } & \multicolumn{2}{|c|}{ Faco ozil } \\
\hline & $\begin{array}{c}\text { Washing } \\
\text { times }\end{array}$ & $\begin{array}{c}\text { Prep \& } \\
\text { pack times }\end{array}$ & $\begin{array}{c}\text { Washing } \\
\text { times }\end{array}$ & $\begin{array}{c}\text { Prep \& } \\
\text { pack times }\end{array}$ & $\begin{array}{c}\text { Washing } \\
\text { times }\end{array}$ & $\begin{array}{c}\text { Prep \& } \\
\text { pack times }\end{array}$ & $\begin{array}{c}\text { Washing } \\
\text { times }\end{array}$ & $\begin{array}{c}\text { Prep \& } \\
\text { pack times }\end{array}$ \\
\hline 1 & 0.40 & 3.57 & 9.15 & 7.47 & 7.40 & 6.50 & 0.37 & 1.01 \\
\hline 2 & 0.41 & 3.42 & 5.29 & 5.28 & 12.36 & 4.41 & 1.11 & 0.42 \\
\hline 3 & 0.41 & 3.23 & 7.38 & 5.20 & 15.43 & 4.25 & 0.58 & 0.58 \\
\hline 4 & 0.55 & 3.04 & 5.31 & 5.35 & 11.17 & 3.21 & 0.43 & 0.59 \\
\hline 5 & 1.08 & 3.18 & 4.12 & 5.04 & 7.51 & 4.06 & 0.50 & 0.39 \\
\hline 6 & 1.09 & 3.28 & 5.17 & 4.00 & 11.09 & 4.18 & 0.52 & 1.05 \\
\hline 7 & 0.56 & 2.21 & 6.29 & 3.22 & 10.48 & 4.22 & 0.56 & 0.34 \\
\hline 8 & 0.45 & 2.32 & 5.03 & 4.51 & 7.39 & 5.09 & 1.27 & 1.05 \\
\hline 9 & 1.08 & 3.24 & 5.53 & 4.30 & 8.06 & 3.15 & 0.35 & 1.19 \\
\hline 10 & 1.16 & 2.38 & 4.02 & 5.24 & 16.26 & 3.29 & 0.41 & 0.35 \\
\hline 11 & 1.03 & 4.19 & 5.37 & 7.15 & 14.16 & 3.53 & 1.05 & 0.37 \\
\hline 12 & 1.13 & 2.46 & 4.27 & 5.26 & 17.59 & 4.57 & 0.30 & 0.53 \\
\hline$\overline{s_{i j}}$ & 0.78 & 3.04 & 5.58 & 5.17 & 11.58 & 4.21 & 0.62 & 0.66 \\
\hline$S$ & 0.33 & 0.59 & 1.46 & 1.19 & 3.64 & 0.94 & 0.33 & 0.32 \\
\hline
\end{tabular}

URL: http://mc.manuscriptcentral.com/tppc E-mail: ppc@plymouth.ac.uk 